Bundesgesundheitsbl - Gesundheitsforsch Gesundheitsschutz 2007 · 50:609-623 DOI 10.1007/s00103-007-0222-x

c) Springer Medizin Verlag 2007

\author{
G. B. M. Mensink · C. Kleiser · A. Richter \\ Robert Koch-Institut, Berlin, BRD
}

\title{
Lebensmittelverzehr bei Kindern und Jugendlichen in Deutschland
}

\author{
Ergebnisse des Kinder- und Jugendgesund- \\ heitssurveys (KiGGS)
}

\section{Hintergrund und Fragestellung}

Die Ernährung ist essenziell für Aufbau und Erhalt der Körperfunktionen. In jeder Lebensphase ist eine ausgewogene Ernährung deshalb für Gesundheit und Wohlbefinden wichtig. Von besonderer Bedeutung ist sie jedoch für die gesundheitliche Entwicklung von Kindern und Jugendlichen. Vor allem in den letzten Jahren rückte die Ernährung dieser Altersgruppe, vor dem Hintergrund der wachsenden Prävalenz von Übergewicht und Adipositas in frühen Lebensjahren, in den Fokus der Gesundheitsvorsorge. Bislang ist das Ernährungsverhalten von Kindern und Jugendlichen in Deutschland aber weitestgehend unbekannt. Bisherige Erkenntnisse stammen vorwiegend aus regionalen Studien, die häufig einen begrenzten Altersbereich oder spezielle Situationen (z. B. die Ernährung in Kindertagesstätten) untersuchten $[1,2,3,4,5,6]$.

Im Rahmen der ersten Nationalen Verzehrsstudie wurden vor etwa 20 Jahren in den alten Bundesländern letztmalig repräsentative Daten zur Lebensmittel- und Nährstoffaufnahme bei Kindern und Jugendlichen ab 4 Jahren erhoben [7]. Die von November 2005 bis Oktober 2006 durchgeführte Nationale Verzehrsstudie II untersuchte lediglich den Altersbereich ab 14 Jahre [8]. Die relativ aktuelle bundes- weite VELS-Studie untersuchte Kinder im Alter von 0-4 Jahre [9].

Seit der ersten Nationalen Verzehrsstudie kann sich viel an den Ernährungsgewohnheiten geändert haben. So hat sich das spezifisch auf Kinder ausgerichtete Lebensmittelangebot, das Spektrum an nährstoffangereicherten Produkten, Convenience-Produkten und Fast Food in den letzten Jahren erheblich erweitert. Internationale Studien deuten darauf hin, dass dieses deutlich erweiterte Lebensmittelangebot vermutlich zu Veränderungen im Lebensmittelkonsum geführt hat. Wahrscheinlich haben sich auch die Mahlzeitengewohnheiten verändert. So gibt es in der internationalen Literatur Hinweise darauf, dass zunehmend das Frühstück ausgelassen, immer seltener gemeinsam mit der Familie gegessen wird und häufiger Snacks verzehrt werden $[10,11,12]$. Die Veränderungen im Lebensmittelangebot sowie der Trend zu einem überwiegend sitzenden Lebensstil können zu einer positiven Energiebilanz beitragen und somit eine Erklärung für die Zunahme von Adipositas im Kindesund Jugendalter sein.

Um eine ausgewogene Ernährung zu erzielen, mit der der Bedarf an essenziellen Nährstoffen gedeckt wird, die aber gleichzeitig nicht zu viel Fett und Zucker enthält, empfehlen das Forschungsinstitut für Kinderernährung in Dortmund (FKE), die Deutsche Gesellschaft für Ernährung (DGE) sowie der Infodienst Verbraucherschutz - Ernährung - Landwirtschaft (aid) eine vielseitige Ernährungsweise, die Lebensmittel aus unterschiedlichen Lebensmittelgruppen enthält. Reichlich sollen kalorienfreie oder -arme Getränke und pflanzliche Lebensmittel (Obst, Gemüse, Getreideprodukte, Reis, Nudeln und Kartoffeln) konsumiert werden. Zudem wird ein mäßiger Verzehr von tierischen Produkten wie Milch und Milchprodukten, Fleisch, Wurst, Fisch und Eiern sowie ein sparsamer Konsum von fett- und zuckerreichen Lebensmitteln (Speisefette, fette Snacks, Knabberartikel und Süßwaren) empfohlen [13, 14, 15].

Der Kinder- und Jugendgesundheitssurvey (KiGGS) kann erstmalig seit langer Zeit für die 1- bis 17-Jährigen ein repräsentatives Bild von Verzehrhäufigkeiten der wichtigsten Lebensmittel in Deutschland zeigen. Diese Informationen können außerdem mit einer Vielzahl weiterer Gesundheitsdaten aus dem Survey verknüpft werden. Allerdings werden die Ernährungsgewohnheiten mit dem in KiGGS eingesetzten Instrument nicht umfassend abgebildet. Die Berechnung von Nährstoffaufnahmen ist nicht und ein Vergleich mit den aktuellen Empfehlungen nur bedingt möglich. Dennoch 
kann ein erster Eindruck vom Ernährungsverhalten der Kinder und Jugendlichen gewonnen werden. Detailliertere und quantitativere Aussagen sind mit der Ernährungsstudie als KiGGS-Modul (EsKiMo) möglich $[16,17]$.

\section{Methoden}

Konzept, Design und Durchführung des KiGGS werden in den ersten 3 Beiträgen in diesem Heft ausführlich beschrieben $[18,19,20]$. Die KiGGS-Studie wurde von Mai 2003 bis Mai 2006 durch das Robert Koch-Institut (RKI) durchgeführt. Ziel dieses bundesweiten Befragungs- und Untersuchungssurveys war es, erstmals umfassende repräsentative Daten zum Gesundheitszustand von Kindern und Jugendlichen im Alter von o-17 Jahren $\mathrm{zu}$ erheben. An der Studie haben insgesamt 17.641 Kinder und Jugendliche (8656 Mädchen und 8985 Jungen) aus 167 für die Bundesrepublik repräsentativen Städten und Gemeinden teilgenommen. Die Teilnahmequote betrug 66,6 \%. Um repräsentative Aussagen treffen zu können, wurden die Analysen mit einem Gewichtungsfaktor durchgeführt, der Abweichungen der Netto-Stichprobe von der Bevölkerungsstruktur (Stand: 31.12.2004) hinsichtlich Alter (in Jahren), Geschlecht, Region (Ost/West/Berlin) und Staatsangehörigkeit korrigiert. Um die Korrelation der Probanden innerhalb einer Gemeinde zu berücksichtigen, wurden die Konfidenzintervalle mit den SPSS-14.0-Verfahren für komplexe Stichproben und mit den Survey-Prozeduren von SAS 9.1 bestimmt [21, 22]. Gruppenunterschiede mit p-Werten von kleiner als 0,05 oder mit 95\%-Konfidenzintervallen, die sich nicht überschneiden, werden als statistisch signifikant gewertet.

Für die Teilnehmer in allen Altersklassen füllten die Eltern Fragebögen zur Gesundheit ihrer Kinder aus, während Kinder und Jugendliche ab 11 Jahren zusätzlich selber über ihr Gesundheitsverhalten Auskunft gaben. Die Kinder und ihre Eltern wurden in den allgemeinen Gesundheitsfragebögen zu gesundheitsrelevanten Themen, wie körperliche und psychische Gesundheit, soziales Umfeld und Lebensbedingungen, Gesundheitsverhalten und medizinische Versorgung,
Übersicht 1

Kurzbezeichnung für im Ernährungsfragebogen abgefragte Lebensmittel (Auswahl)

Kurzbezeichnung Genaue Bezeichnung im Fragebogen

Softdrinks

Energydrinks

Säfte

Leitungswasser

Mineralwasser

Früchtetee

Schwarzer Tee

Kaffee

Milch

Käse

Milchprodukte

Frischkäse

Fleisch

Geflügel

Wurst

Erfrischungsgetränke (z. B. Cola, Limonade, Eistee, Malzbier)

Sportler- oder Energiegetränke

Fruchtsaft, Fruchtnektar, Gemüsesaft (auch verdünnt)

Leitungswasser (auch selbst zubereitetes Sodawasser)

Mineralwasser (ohne Leitungswasser)

Früchte- oder Kräutertee

Schwarzer oder grüner Tee

Kaffee

Milch (einschließlich Kakao, Milch für Müsli)

Käse (Weich-, Schnitt- oder Hartkäse)

Quark, Joghurt oder Dickmilch

Frischkäse

Fleisch (ohne Geflügel, ohne Wurst)

Geflügel

Wurst oder Schinken

Fisch

Weißbrot

Fisch

Vollkornbrot

Cerealien

Obst

Konservenobst

Gemüse, gegart

Tiefkühlgemüse

Konservengemüse

Gemüse, roh

Nudeln, Reis

Kartoffeln

Pommes

Weißbrot, Graubrot oder Brötchen

Vollkornbrot, Vollkornbrötchen oder Schwarzbrot

Müsli, Cornflakes oder Smacks

Frisches Obst

Gekochtes Obst oder Konservenobst

Gekochtes Gemüse (aus frischem Gemüse zubereitet)

Tiefkühlgemüse

Konservengemüse

Blattsalat, Rohkost oder rohes Gemüse (z. B. Tomaten, Möhren, Paprika)

Nudeln oder Reis

Gekochte Kartoffeln (Salz-, Pellkartoffeln, Kartoffelbrei, Kartoffelklöße)

Frittierte oder gebratene Kartoffeln (Pommes frites, Kroketten, Bratkartoffeln)

Fast Food Bratwurst, Currywurst, Hamburger, Döner Kebab

Kuchen Kuchen oder Gebäck (ohne Kekse)

Kekse

Schokolade Schokolade oder Schokoriegel

Süßigkeiten

Knabberartikel

Süßigkeiten (z. B. Bonbons oder Fruchtgummi)

Knabberartikel (Chips, Salzstangen, Cracker) befragt [23]. Ein Teil dieser Fragen betraf Aspekte der Ernährung wie das Stillverhalten der Mutter, besondere Ernährungsformen und eventuelle Essstörungen. AuBerdem wurden bestimmte biochemische Parameter zum Ernährungszustand im Blut und Urin ermittelt.

Zusätzlich wurden retrospektiv die durchschnittlichen Verzehrshäufigkeiten und Portionsmengen ,in den letzten Wochen" von etwa 50 Lebensmittelgruppen mit einem am RKI neu entwickelten Ernährungsfragebogen (Food Frequency Questionnaire, FFQ) erfasst, wobei Antwortkategorien sowohl für die Häufigkeit als auch für die Portionsmenge der ein- zelnen Lebensmittel vorgegeben waren [24]. Der Fragebogen lag in 2 Varianten vor. Eltern von Kindern im Alter von 1-10 Jahren füllten den Fragebogen „Was isst Ihr Kind?" aus, während Jugendliche im Alter von 11-17 Jahren den Fragebogen „Was isst du?" beantworteten. Die hiermit erfassten Variablen sind jedoch deckungsgleich. Die Antwortkategorien für die Verzehrshäufigkeiten waren für alle Lebensmittelgruppen in gleicher Weise angegeben („Nie“, „einmal im Monat“, „2- bis 3-mal im Monat“, „1- bis 2-mal pro Woche", „3- bis 4-mal pro Woche“, "5- bis 6-mal pro Woche“, „einmal am Tag“, „2bis 3-mal am Tag“, "4- bis 5-mal am Tag“, 


\section{Zusammenfassung $\cdot$ Abstract}

Bundesgesundheitsbl - Gesundheitsforsch - Gesundheitsschutz 2007 · 50:609-623

DOI 10.1007/s00103-007-0222-x

(C) Springer Medizin Verlag 2007

\section{G. B.M. Mensink · C. Kleiser · A. Richter}

\section{Lebensmittelverzehr bei Kindern und Jugendlichen in Deutschland. Ergebnisse des Kinder- und Jugendgesundheitssurveys (KiGGS)}

\section{Zusammenfassung}

Da das Ernährungsverhalten einen wesentlichen Einfluss auf die Gesundheit hat, wurden im Rahmen des Kinder- und Jugendgesundheitssurveys (KiGGS) auch Informationen zum Lebensmittelverzehr erfasst. Mit Hilfe eines Ernährungsfragebogens wurden die Verzehrshäufigkeiten und Portionsmengen verschiedener Lebensmittelgruppen „in den letzten Wochen" ermittelt. Zwei inhaltlich identische Varianten des Fragebogens, einer für die Eltern der 1- bis 10-Jährigen und einer für die Befragten im Alter von 11-17 Jahren, wurden verwendet. Die Ergebnisse beruhen auf Verzehrsdaten von 7186 Jungen und 6919 Mädchen im Alter von 3-17 Jahren. Etwa die
Hälfte der Teilnehmer konsumiert jeweils täglich Brot, Milch und frisches Obst. Die Mehrheit (etwa drei Viertel) isst lediglich einmal im Monat Fast Food. Jedoch essen täglich 16\% Schokolade und fast $20 \%$ andere Süßigkeiten. Mehr Jungen als Mädchen konsumieren jeweils täglich Erfrischungsgetränke, Milch, Milchprodukte, Fleisch, Wurst, Cerealien und Weißbrot. Dafür essen Mädchen häufiger täglich frisches Obst und rohes Gemüse. Mit steigendem Alter nimmt der Anteil derjenigen, die täglich Erfrischungsgetränke, Käse (und bei Jungen auch Fleisch und Wurst) konsumieren, zu. Der Anteil derjenigen, die täglich Säfte, Leitungswasser, Milch, Milch- produkte, frisches Obst, rohes Gemüse, Kekse, Süßigkeiten, Schokolade (und bei Mädchen außerdem Frühstückscerealien) konsumieren, nimmt hingegen ab. Wie erwartet ist der Konsum von Süßigkeiten und süßen Getränken relativ hoch. Während mehr als die Hälfte der Kinder täglich Obst bzw. Gemüse konsumiert, nimmt dieser Anteil mit zunehmendem Alter ab.

\section{Schlüsselwörter}

Gesundheitssurvey · Kinder · Jugendliche · Ernährungsepidemiologie · Verzehrshäufigkeitsfragebogen

\section{Food consumption of children and adolescents in Germany. Results of the German Health Interview and Examination Survey for Children and Adolescents (KiGGS)}

\section{Abstract}

Since dietary behaviour has an essential impact on health, information on food consumption was obtained in the German Health Interview and Examination Survey for Children and Adolescents (KiGGS). The average food frequency and portion size "in recent weeks" was assessed with a food frequency questionnaire. Two versions of the questionnaire, identical in content, exist: one aimed at parents of 1-10-year-olds and the other directly at 11-17-year-olds. The results include data on 7,186 boys and 6,919 girls, aged 3-17. About half of the participants each consume bread, milk and fresh fruits every day. The majority (about three quarter) eat fast food only once a month or less. However, $16 \%$ of the participants eat chocolate, and almost $20 \%$ other sweets every day. More boys than girls individually consume soft drinks, milk, dairy products, meat, sausage, cereals and white bread every day. More girls consume fresh fruits and raw vegetables each day. With increasing age the proportion of daily consumers of soft drinks, cheese (and among boys also meat and sausage) increases, but the proportion of daily consumers of juices, tap water, milk, dairy products, fresh fruits, raw vegetables, cookies, chocolate, sweets (and among girls also breakfast cereals) falls. As expected the consumption of sweets and soft drinks is relatively high. Whereas more than half of the children each consume fruits and vegetables on a daily base, this consumption declines with increasing age.

\section{Keywords}

Health survey · Children · Adolescents . Nutritional epidemiology · Dietary assessment methods 
Tabelle 1

Verzehrshäufigkeiten von ausgewählten Lebensmitteln nach Geschlecht (Prozent und $95 \%$-Konfidenzintervall)

\begin{tabular}{|c|c|c|c|c|c|c|c|c|c|c|c|}
\hline \multirow[t]{3}{*}{ Lebensmittel } & \multirow[t]{3}{*}{ Geschlecht } & \multicolumn{10}{|c|}{ Verzehrshäufigkeiten } \\
\hline & & \multicolumn{2}{|c|}{ Nie } & \multicolumn{2}{|c|}{$\begin{array}{l}\text { 1- bis 3-mal/ } \\
\text { Monat }\end{array}$} & \multicolumn{2}{|c|}{$\begin{array}{l}\text { 1- bis 6-mal/ } \\
\text { Woche }\end{array}$} & \multicolumn{2}{|c|}{ Täglich } & \multicolumn{2}{|c|}{$\begin{array}{l}\text { Mehrmals } \\
\text { Täglich }\end{array}$} \\
\hline & & $\%$ & (95\% KI) & $\%$ & (95\% KI) & $\%$ & $(95 \% \mathrm{KI})$ & $\%$ & (95\% KI) & $\%$ & (95\% KI) \\
\hline \multirow[t]{2}{*}{ Softdrinks } & Jungen & 8,4 & $(7,5-9,3)$ & 26,8 & $(25,5-28,1)$ & 30,8 & $(29,6-32,1)$ & 8,8 & $(7,9-9,6)$ & 25,2 & $(23,7-26,7)$ \\
\hline & Mädchen & 10,8 & $(9,9-11,8)$ & 32,0 & $(30,3-33,6)$ & 29,0 & $(27,7-30,4)$ & 8,4 & $(7,6-9,3)$ & 19,8 & $(18,4-21,1)$ \\
\hline \multirow[t]{2}{*}{ Energydrinks } & Jungen & 66,0 & $(64,4-67,5)$ & 23,5 & $(22,2-24,7)$ & 8,2 & $(7,4-8,9)$ & 1,6 & $(1,2-2,0)$ & 0,7 & $(0,5-1,0)$ \\
\hline & Mädchen & 75,6 & $(74,2-77,0)$ & 17,9 & $(16,7-19,1)$ & 5,0 & $(4,4-5,7)$ & 1,0 & $(0,7-1,2)$ & 0,6 & $(0,4-0,8)$ \\
\hline \multirow[t]{2}{*}{ Säfte } & Jungen & 5,5 & $(4,9-6,1)$ & 13,1 & $(12,1-14,1)$ & 30,8 & $(29,5-32,1)$ & 15,5 & $(14,4-16,5)$ & 35,2 & $(33,5-36,9)$ \\
\hline & Mädchen & 4,6 & $(4,1-5,2)$ & 13,9 & $(12,8-15,0)$ & 30,9 & $(29,7-32,1)$ & 14,6 & $(13,6-15,5)$ & 36,0 & $(34,3-37,8)$ \\
\hline \multirow[t]{2}{*}{ Leitungswasser } & Jungen & 43,8 & $(41,7-45,9)$ & 13,9 & $(12,9-14,8)$ & 14,0 & $(13,0-15,1)$ & 8,5 & $(7,7-9,3)$ & 19,9 & $(18,3-21,5)$ \\
\hline & Mädchen & 42,5 & $(40,3-44,7)$ & 13,5 & $(12,6-14,4)$ & 12,9 & $(11,9-13,9)$ & 9,0 & $(8,1-9,9)$ & 22,1 & $(20,3-23,8)$ \\
\hline \multirow[t]{2}{*}{ Mineralwasser } & Jungen & 13,6 & $(12,4-14,8)$ & 13,7 & $(12,5-14,9)$ & 22,5 & $(21,3-23,7)$ & 11,3 & $(10,5-12,1)$ & 38,9 & $(36,8-41,0)$ \\
\hline & Mädchen & 12,8 & $(11,5-14,0)$ & 12,3 & $(11,2-13,3)$ & 20,7 & $(19,6-21,8)$ & 11,3 & $(10,4-12,2)$ & 42,9 & $(40,8-45,1)$ \\
\hline \multirow[t]{2}{*}{ Früchtetee } & Jungen & 33,2 & $(31,0-35,4)$ & 25,8 & $(24,6-27,0)$ & 22,8 & $(21,4-24,2)$ & 11,4 & $(10,4-12,5)$ & 6,8 & $(6,0-7,6)$ \\
\hline & Mädchen & 26,4 & $(24,4-28,4)$ & 26,3 & $(25,1-27,5)$ & 26,0 & $(24,7-27,4)$ & 13,2 & $(12,1-14,3)$ & 8,1 & $(7,2-8,9)$ \\
\hline \multirow[t]{2}{*}{ Schwarzer Tee } & Jungen & 78,1 & $(75,9-80,2)$ & 10,3 & $(9,4-11,3)$ & 6,9 & $(5,8-7,9)$ & 3,2 & $(2,5-3,9)$ & 1,5 & $(1,1-1,8)$ \\
\hline & Mädchen & 78,4 & $(76,2-80,6)$ & 10,4 & $(9,5-11,3)$ & 6,6 & $(5,6-7,6)$ & 2,9 & $(2,2-3,6)$ & 1,7 & $(1,3-2,2)$ \\
\hline \multirow[t]{2}{*}{ Kaffee } & Jungen & 82,4 & $(81,1-83,6)$ & 8,7 & $(7,9-9,5)$ & 6,0 & $(5,3-6,6)$ & 1,8 & $(1,5-2,2)$ & 1,1 & $(0,8-1,4)$ \\
\hline & Mädchen & 80,8 & $(79,6-82,1)$ & 9,6 & $(8,7-10,4)$ & 5,9 & $(5,3-6,6)$ & 2,5 & $(2,1-2,9)$ & 1,1 & $(0,8-1,4)$ \\
\hline \multirow[t]{2}{*}{ Milch } & Jungen & 3,9 & $(3,4-4,5)$ & 8,1 & $(7,3-8,9)$ & 32,6 & $(31,4-33,9)$ & 32,0 & $(30,7-33,3)$ & 23,4 & $(22,1-24,6)$ \\
\hline & Mädchen & 3,5 & $(2,9-4,0)$ & 10,7 & $(9,7-11,6)$ & 36,1 & $(34,7-37,5)$ & 31,5 & $(30,1-32,8)$ & 18,3 & $(17,0-19,6)$ \\
\hline \multirow[t]{2}{*}{ Käse } & Jungen & 16,4 & $(15,3-17,5)$ & 13,9 & $(13,0-14,9)$ & 46,8 & $(45,4-48,2)$ & 16,2 & $(15,1-17,3)$ & 6,7 & $(5,9-7,4)$ \\
\hline & Mädchen & 12,1 & $(11,2-12,9)$ & 13,6 & $(12,7-14,6)$ & 50,3 & $(48,9-51,7)$ & 16,6 & $(15,4-17,7)$ & 7,4 & $(6,6-8,2)$ \\
\hline \multirow[t]{2}{*}{ Milchprodukte } & Jungen & 8,4 & $(7,5-9,2)$ & 14,9 & $(14,0-15,9)$ & 50,5 & $(49,1-51,9)$ & 20,7 & $(19,6-21,7)$ & 5,5 & $(4,9-6,1)$ \\
\hline & Mädchen & 5,7 & $(5,1-6,4)$ & 16,8 & $(15,9-17,8)$ & 54,8 & $(53,4-56,1)$ & 19,0 & $(17,9-20,1)$ & 3,7 & $(3,2-4,2)$ \\
\hline Frischkäse & Jungen & 40,4 & $(38,9-41,9)$ & 26,3 & $(25,1-27,6)$ & 27,7 & $(26,3-29,0)$ & 4,4 & $(3,8-4,9)$ & 1,2 & $(0,9-1,5)$ \\
\hline & Mädchen & 32,9 & $(31,5-34,3)$ & 27,6 & $(26,4-28,9)$ & 32,9 & $(31,5-34,4)$ & 5,3 & $(4,6-6,0)$ & 1,3 & $(1,0-1,7)$ \\
\hline Fleisch & Jungen & 3,2 & $(2,7-3,7)$ & 17,0 & $(15,9-18,2)$ & 73,2 & $(71,9-74,5)$ & 5,3 & $(4,6-6,0)$ & 1,3 & $(1,0-1,6)$ \\
\hline & Mädchen & 5,9 & $(5,2-6,6)$ & 22,3 & $(21,1-23,6)$ & 67,3 & $(65,9-68,7)$ & 3,7 & $(3,1-4,4)$ & 0,7 & $(0,5-1,0)$ \\
\hline Geflügel & Jungen & 4,2 & $(3,7-4,7)$ & 46,1 & $(44,1-48,2)$ & 48,8 & $(46,8-50,9)$ & 0,7 & $(0,5-0,9)$ & 0,2 & $(0,1-0,3)$ \\
\hline & Mädchen & 6,1 & $(5,5-6,8)$ & 46,8 & $(45,0-48,5)$ & 46,1 & $(44,4-47,9)$ & 0,8 & $(0,6-1,1)$ & 0,1 & $(0,0-0,3)$ \\
\hline Wurst & Jungen & 4,1 & $(3,5-4,7)$ & 7,6 & $(6,8-8,5)$ & 48,3 & $(47,0-49,5)$ & 28,4 & $(27,2-29,5)$ & 11,6 & $(10,8-12,5)$ \\
\hline & Mädchen & 6,2 & $(5,4-6,9)$ & 10,2 & $(9,3-11,2)$ & 52,0 & $(50,5-53,6)$ & 23,1 & $(21,9-24,4)$ & 8,4 & $(7,6-9,2)$ \\
\hline Fisch & Jungen & 13,2 & $(12,3-14,2)$ & 58,5 & $(57,1-60,0)$ & 27,7 & $(26,5-28,9)$ & 0,4 & $(0,3-0,6)$ & 0,1 & $(0,0-0,2)$ \\
\hline & Mädchen & 16,0 & $(14,9-17,0)$ & 59,4 & $(57,9-60,8)$ & 24,3 & $(23,0-25,6)$ & 0,3 & $(0,1-0,4)$ & 0,1 & $(0,0-0,1)$ \\
\hline Cerealien & Jungen & 10,1 & $(9,2-11,1)$ & 20,6 & $(19,4-21,8)$ & 43,5 & $(41,9-45,1)$ & 22,3 & $(21,2-23,4)$ & 3,5 & $(3,0-4,1)$ \\
\hline & Mädchen & 9,9 & $(9,1-10,8)$ & 23,8 & $(22,4-25,1)$ & 44,0 & $(42,5-45,6)$ & 19,2 & $(18,1-20,2)$ & 3,1 & $(2,6-3,6)$ \\
\hline Vollkornbrot & Jungen & 15,8 & $(14,5-17,0)$ & 15,4 & $(14,4-16,5)$ & 35,5 & $(34,1-36,9)$ & 18,0 & $(16,8-19,3)$ & 15,3 & $(14,1-16,4)$ \\
\hline & Mädchen & 12,1 & $(11,2-13,1)$ & 15,9 & $(14,9-17,0)$ & 36,6 & $(35,3-38,0)$ & 19,6 & $(18,4-20,7)$ & 15,8 & $(14,7-16,8)$ \\
\hline Weißbrot & Jungen & 1,3 & $(1,0-1,6)$ & 6,4 & $(5,7-7,1)$ & 46,9 & $(45,5-48,3)$ & 24,3 & $(23,2-25,5)$ & 21,1 & $(19,7-22,6)$ \\
\hline & Mädchen & 1,2 & $(0,9-1,4)$ & 8,1 & $(7,3-8,9)$ & 51,4 & $(49,8-53,0)$ & 22,9 & $(21,8-24,1)$ & 16,4 & $(15,2-17,7)$ \\
\hline Obst & Jungen & 2,3 & $(1,9-2,7)$ & 9,7 & $(8,9-10,5)$ & 40,8 & $(39,4-42,2)$ & 29,4 & $(28,1-30,6)$ & 17,8 & $(16,6-19,0)$ \\
\hline & Mädchen & 0,9 & $(0,6-1,1)$ & 6,6 & $(5,9-7,3)$ & 38,1 & $(36,6-39,6)$ & 30,4 & $(29,2-31,6)$ & 24,1 & $(22,7-25,5)$ \\
\hline Gemüse gegart & Jungen & 11,3 & $(10,3-12,3)$ & 25,2 & $(23,8-26,5)$ & 57,2 & $(55,8-58,7)$ & 5,9 & $(5,2-6,6)$ & 0,4 & $(0,2-0,5)$ \\
\hline & Mädchen & 9,8 & $(9,0-10,6)$ & 24,6 & $(23,4-25,9)$ & 58,7 & $(57,3-60,0)$ & 6,5 & $(5,8-7,3)$ & 0,3 & $(0,2-0,5)$ \\
\hline Tiefkühlgemüse & Jungen & 26,2 & $(24,8-27,6)$ & 41,1 & $(39,5-42,6)$ & 31,8 & $(30,3-33,4)$ & 0,8 & $(0,6-1,1)$ & 0,1 & $(0,0-0,1)$ \\
\hline & Mädchen & 23,9 & $(22,5-25,2)$ & 43,0 & $(41,4-44,6)$ & 32,5 & $(30,7-34,3)$ & 0,6 & $(0,4-0,8)$ & 0,1 & $(0,0-0,1)$ \\
\hline Konservengemüse & Jungen & 39,3 & $(37,4-41,1)$ & 42,8 & $(41,5-44,1)$ & 17,5 & $(15,9-19,1)$ & 0,4 & $(0,2-0,6)$ & 0,0 & $(0,0-0,1)$ \\
\hline & Mädchen & 37,9 & $(36,0-39,7)$ & 44,6 & $(43,1-46,2)$ & 17,1 & $(15,5-18,7)$ & 0,4 & $(0,2-0,5)$ & 0,0 & $(0,0-0,1)$ \\
\hline
\end{tabular}


Tabelle 1 (Fortsetzung)

\begin{tabular}{|c|c|c|c|c|c|c|c|c|c|c|c|}
\hline \multirow[t]{3}{*}{ Lebensmittel } & \multirow[t]{3}{*}{ Geschlecht } & \multicolumn{10}{|c|}{ Verzehrshäufigkeiten } \\
\hline & & \multicolumn{2}{|l|}{ Nie } & \multicolumn{2}{|c|}{$\begin{array}{l}\text { 1- bis 3-mal/ } \\
\text { Monat }\end{array}$} & \multicolumn{2}{|c|}{$\begin{array}{l}\text { 1- bis 6-mal/ } \\
\text { Woche }\end{array}$} & \multicolumn{2}{|c|}{ Täglich } & \multicolumn{2}{|c|}{$\begin{array}{l}\text { Mehrmals } \\
\text { Täglich }\end{array}$} \\
\hline & & $\%$ & (95\% KI) & $\%$ & (95\% KI) & $\%$ & (95\% KI) & $\%$ & (95\% KI) & $\%$ & (95\% KI) \\
\hline \multirow[t]{2}{*}{ Gemüse roh } & Jungen & 8,2 & $(7,3-9,1)$ & 15,6 & $(14,5-16,7)$ & 54,8 & $(53,5-56,0)$ & 17,7 & $(16,6-18,7)$ & 3,7 & $(3,2-4,3)$ \\
\hline & Mädchen & 3,8 & $(3,2-4,4)$ & 12,4 & $(11,3-13,4)$ & 55,3 & $(53,9-56,8)$ & 22,6 & $(21,2-24,0)$ & 5,9 & $(5,2-6,5)$ \\
\hline \multirow[t]{2}{*}{ Nudeln, Reis } & Jungen & 0,3 & $(0,1-0,4)$ & 11,0 & $(10,0-12,1)$ & 84,5 & $(83,4-85,6)$ & 3,4 & $(2,8-4,1)$ & 0,8 & $(0,5-1,0)$ \\
\hline & Mädchen & 0,1 & $(0,0-0,2)$ & 10,3 & $(9,3-11,4)$ & 85,1 & $(84,1-86,2)$ & 3,7 & $(3,0-4,3)$ & 0,7 & $(0,5-1,0)$ \\
\hline \multirow[t]{2}{*}{ Kartoffeln } & Jungen & 2,3 & $(1,9-2,7)$ & 15,7 & $(14,1-17,2)$ & 78,7 & $(77,1-80,3)$ & 3,0 & $(2,4-3,6)$ & 0,3 & $(0,2-0,5)$ \\
\hline & Mädchen & 1,7 & $(1,3-2,1)$ & 15,6 & $(14,2-17,0)$ & 79,8 & $(78,4-81,2)$ & 2,6 & $(2,1-3,2)$ & 0,3 & $(0,1-0,4)$ \\
\hline \multirow[t]{2}{*}{ Pommes } & Jungen & 4,6 & $(4,0-5,2)$ & 65,0 & $(63,5-66,5)$ & 29,7 & $(28,0-31,3)$ & 0,6 & $(0,4-0,8)$ & 0,1 & $(0,0-0,2)$ \\
\hline & Mädchen & 6,3 & $(5,3-7,2)$ & 68,6 & $(67,0-70,2)$ & 24,6 & $(23,0-26,2)$ & 0,3 & $(0,2-0,5)$ & 0,2 & $(0,0-0,3)$ \\
\hline \multirow[t]{2}{*}{ Fast Food } & Jungen & 9,2 & $(8,4-9,9)$ & 72,3 & $(71,0-73,5)$ & 18,0 & $(16,8-19,3)$ & 0,5 & $(0,3-0,7)$ & 0,0 & $(0,0-0,1)$ \\
\hline & Mädchen & 15,4 & $(14,1-16,7)$ & 75,1 & $(73,7-76,5)$ & 9,1 & $(8,1-10,0)$ & 0,4 & $(0,2-0,6)$ & 0,0 & $(0,0-0,1)$ \\
\hline \multirow[t]{2}{*}{ Kuchen } & Jungen & 4,1 & $(3,5-4,7)$ & 48,3 & $(46,6-50,1)$ & 44,0 & $(42,1-45,8)$ & 3,2 & $(2,7-3,7)$ & 0,4 & $(0,2-1,6)$ \\
\hline & Mädchen & 3,7 & $(3,2-4,3)$ & 50,6 & $(48,7-52,5)$ & 42,5 & $(40,6-44,3)$ & 2,8 & $(2,4-3,3)$ & 0,4 & $(0,2-0,5)$ \\
\hline \multirow[t]{2}{*}{ Kekse } & Jungen & 8,4 & $(7,6-9,2)$ & 37,8 & $(36,5-39,2)$ & 47,7 & $(46,3-49,1)$ & 4,9 & $(4,3-5,5)$ & 1,3 & $(0,9-1,6)$ \\
\hline & Mädchen & 7,9 & $(7,0-8,7)$ & 40,2 & $(38,7-41,8)$ & 46,0 & $(44,2-47,7)$ & 4,8 & $(4,2-5,4)$ & 1,2 & $(0,9-1,5)$ \\
\hline \multirow[t]{2}{*}{ Schokolade } & Jungen & 2,9 & $(2,5-3,3)$ & 20,8 & $(19,7-21,9)$ & 60,5 & $(59,2-61,8)$ & 12,1 & $(11,2-13,0)$ & 3,6 & $(3,1-4,2)$ \\
\hline & Mädchen & 2,8 & $(2,3-3,2)$ & 23,0 & $(21,9-24,2)$ & 58,4 & $(57,1-59,6)$ & 12,4 & $(11,4-13,3)$ & 3,4 & $(2,9-3,9)$ \\
\hline \multirow[t]{2}{*}{ Süßigkeiten } & Jungen & 3,7 & $(3,2-4,2)$ & 19,5 & $(18,4-20,6)$ & 57,6 & $(56,3-58,8)$ & 14,3 & $(13,2-15,3)$ & 5,0 & $(4,4-5,6)$ \\
\hline & Mädchen & 3,1 & $(2,6-3,6)$ & 19,2 & $(18,2-20,3)$ & 58,0 & $(56,6-59,5)$ & 14,7 & $(13,7-15,8)$ & 4,9 & $(4,2-5,5)$ \\
\hline \multirow[t]{2}{*}{ Knabberartikel } & Jungen & 7,6 & $(6,9-8,4)$ & 53,5 & $(52,1-54,9)$ & 36,1 & $(34,5-37,7)$ & 2,1 & $(1,8-2,5)$ & 0,6 & $(0,4-0,9)$ \\
\hline & Mädchen & 9,6 & $(8,7-10,6)$ & 56,6 & $(55,0-58,2)$ & 31,6 & $(30,0-33,2)$ & 1,6 & $(1,3-2,0)$ & 0,5 & $(0,3-0,7)$ \\
\hline
\end{tabular}

„Öfter als 5-mal am Tag“). Die Portionsmengen wurden je nach Lebensmittel unterschiedlich erfragt, z. B. „1/4 Portion (oder weniger) “, „1/2 Portion“, „1 Portion“, „2 Portionen“, „3 Portionen (oder mehr)“, wobei für alle Lebensmittelitems 5 Kategorien vorgegeben waren. Als Hilfestellung zur Einschätzung der erfragten Portionsmengen sind viele beispielhaft in Bildern dargestellt. Zudem wurde nach der Konsumhäufigkeit von Supplementen und angereicherten Lebensmitteln, LightProdukten, Fertiggerichten und probiotischen Lebensmitteln gefragt. Beide Fragebogenversionen sind unter http://www. kiggs.de abrufbar.

Wegen des Umfangs des gesamten erfragten Spektrums werden nachfolgend lediglich besonders relevante Lebensmittel dargestellt. Außerdem werden verkürzte Begriffe für die Lebensmittelgruppen verwendet, die dazugehörigen genauen Abfrageformulierungen sind in der Übersicht 1 enthalten. Der Lebensmittelverzehr wurde nach Geschlechts- und Altersgruppen und nach Migrationshintergrund [25] ausgewertet. Die o- bis 2-jährigen Kinder wurden hier nicht berücksichtigt, da die- se Altergruppe teilweise noch gestillt wird oder Beikost erhält bzw. bereits vollständig an Familienmahlzeiten teilnimmt. Aus diesem Grund ist diese Altergruppe hinsichtlich ihrer Verzehrsgewohnheiten schwer zusammenzufassen und mit den anderen zu vergleichen. Aus Platzgründen wurde auf eine weitere Aufteilung nach sozialem Status verzichtet, dies ist für eine nächste Publikation vorgesehen.

\section{Ergebnisse}

Von den 16.706 über einjährigen KiGGSTeilnehmern liegen für 15.945 ausgefüllte Ernährungsfragebögen vor (95\%). Einige Fragebögen, die offensichtlich nicht vollständig ausgefüllt wurden, und einige mit insgesamt unplausibel hohen Lebensmittelverzehrshäufigkeiten und Mengen wurden für die Auswertungen ausgeschlossen. Somit sind für 15.865 Teilnehmer (8073 Jungen und 7792 Mädchen) Ernährungsdaten vorhanden. Die hier aufgeführten Ergebnisse beruhen auf den Teilnehmern ab einem Alter von 3 Jahren - insgesamt 7186 Jungen und 6919 Mädchen.
Da die Gruppe der Kinder und Jugendlichen mit Migrationshintergrund, was die Esskultur betrifft, eine sehr heterogene Gruppe ist, wurde sie für die Auswertung in zwei Hauptgruppen eingeteilt: türkische Migranten und deutschstämmige Aussiedler. Darüber hinaus sind 2 weitere Gruppen aufgeführt: sonstige Migranten (d.h. anderer Migrationshintergrund) und Nicht-Migranten. Insgesamt haben 276 Jungen aus türkischen, 289 aus deutschstämmigen und 413 aus sonstigen Migrantenfamilien und 201 Mädchen aus türkischen, 288 aus deutschstämmigen und 395 aus sonstigen Migrantenfamilien im Alter ab 3 Jahren bzw. deren Eltern den Ernährungsfragebogen ausgefüllt.

In - Tabelle 1 sind für ausgewählte Lebensmittelgruppen die Verzehrshäufigkeiten und $95 \%$-Konfidenzintervalle (95\%-KI) für Mädchen und Jungen dargestellt. Dafür wurden die 10 Häufigkeitskategorien auf 5 aggregiert.• Tabelle 2 umfasst die Prävalenz (und 95\%-KI) von jeweils einmal und mehrmals täglichem Konsum für selektierte Lebensmittelgruppen nach Geschlechts- und Altersklassen. In $\bullet$ Tabelle 3 ist die Prävalenz (und 95\%- 
Tabelle 2

Anteile mit täglichem Konsum von Lebensmitteln nach Geschlecht und Altersklassen (Prozent und 95\%-Konfidenzintervall)

\begin{tabular}{|c|c|c|c|c|c|c|c|c|c|c|c|}
\hline \multirow[t]{3}{*}{ Lebensmittel } & \multirow{3}{*}{ Alter } & \multicolumn{5}{|c|}{ Jungen } & \multicolumn{5}{|c|}{ Mädchen } \\
\hline & & \multicolumn{2}{|c|}{ 3-6 Jahre } & 7-10 Jahre & 11-13 Jahre & 14-17 Jahre & \multicolumn{2}{|c|}{ 3-6 Jahre } & 7-10 Jahre & 11-13 Jahre & 14-17 Jahre \\
\hline & & $\%$ & $(95 \% \mathrm{KI})$ & $(95 \% \mathrm{KI})$ & $(95 \% \mathrm{KI})$ & $(95 \% \mathrm{KI})$ & $\%$ & $(95 \% \mathrm{KI})$ & $(95 \% \mathrm{KI})$ & $(95 \% \mathrm{KI})$ & $(95 \% \mathrm{KI})$ \\
\hline \multirow[t]{2}{*}{ Softdrinks } & Einmal $^{a}$ & 7,0 & $(5,6-8,5)$ & $8,7 \quad(7,4-10,1)$ & $8,3 \quad(6,7-10,0)$ & $(8,9-12,1)$ & 6,4 & $(4,9-8,0)$ & $9,6 \quad(7,9-11,3)$ & $8,5 \quad(6,8-10,3)$ & $9,0 \quad(7,5-10,6)$ \\
\hline & Mehrmals $^{\text {b }}$ & 14,0 & $(12,2-15,9)$ & 22,0 & $26,2(23,4-29,0)$ & 36,0 & 14,1 & $(12,2-16,0)$ & $18,2(15,7-20,7)$ & $21,4(18,5-24,3)$ & $24,3 \quad(22,2-26,4)$ \\
\hline \multirow[t]{2}{*}{ Säfte } & Einmal & 11,7 & $(10,1-13,2)$ & $18,1(16,3-20,0)$ & $15,1 \quad(13,1-17,1)$ & 16,5 & 13,6 & $(11,8-15,4)$ & 16,4 & $15,6(13,5-17,8)$ & $13,2(11,4-14,9)$ \\
\hline & Mehrmals & 54,4 & $(51,6-57,3)$ & $38,7(36,0-41,5)$ & $28,5 \quad(25,7-31,3)$ & $21,2(18,7-23,6)$ & 49,5 & $(46,8-52,3)$ & $37,5(34,7-40,2)$ & $30,4 \quad(27,2-33,6)$ & $27,7 \quad(25,3-30,2)$ \\
\hline \multirow[t]{2}{*}{ Leitungswasser } & Einmal & 12,4 & $(10,7-14,1)$ & $9,0 \quad(7,7-10,3)$ & $6,6 \quad(5,1-8,0)$ & $6,1 \quad(4,8-7,4)$ & 12,1 & $(10,1-14,1)$ & $9,5 \quad(7,9-11,1)$ & $7,4 \quad(5,8-9,0)$ & $7,3 \quad(6,1-8,5)$ \\
\hline & Mehrmals & 23,4 & $(20,6-26,1)$ & $21,7(19,5-24,0)$ & $18,6(16,2-21,1)$ & $16,3(14,3-18,3)$ & 24,2 & $(21,6-26,9)$ & $22,3(19,6-25,0)$ & $20,6(18,0-23,2)$ & $21,2(18,5-23,8)$ \\
\hline \multirow[t]{2}{*}{ Mineralwasser } & Einmal & 13,2 & $(11,5-15,0)$ & $13,3(11,5-15,1)$ & $9,3 \quad(7,6-11,0)$ & $9,4 \quad(8,1-10,7)$ & 14,2 & $(12,3-16,0)$ & $11,5 \quad(9,9-13,1)$ & $11,3 \quad(9,4-13,2)$ & $9,0 \quad(7,4-10,6)$ \\
\hline & Mehrmals & 38,6 & $(35,6-41,7)$ & $39,4(36,5-42,4)$ & $39,5 \quad(36,4-42,7)$ & $38,2(35,5-41,0)$ & 38,6 & $(35,4-41,8)$ & $41,4(38,1-44,8)$ & $44,1 \quad(40,8-47,4)$ & $46,7 \quad(43,8-49,6)$ \\
\hline \multirow[t]{2}{*}{ Milch } & Einmal & 32,7 & $(30,2-35,1)$ & $38,6(36,2-40,9)$ & $31,4(28,8-34,1)$ & 26,2 & 36,5 & $(33,9-39,0)$ & $37,6(34,9-40,2)$ & $28,8(26,1-31,5)$ & $24,3 \quad(22,1-26,6)$ \\
\hline & Mehrmals & 29,7 & $(27,2-32,1)$ & $25,5(23,3-27,6)$ & $21,1(18,7-23,4)$ & $18,1 \quad(16,1-20,2)$ & 24,9 & $(22,2-27,5)$ & $19,1(16,8-21,4)$ & $16,3(14,0-18,7)$ & $13,7(11,5-15,8)$ \\
\hline \multirow[t]{2}{*}{ Käse } & Einmal & 15,3 & $(13,3-17,2)$ & $15,2(13,4-16,9)$ & $15,1 \quad(13,0-17,2)$ & $18,4(16,5-20,4)$ & 17,2 & $(15,1-19,4)$ & $16,2(14,2-18,1)$ & $14,8(12,6-17,0)$ & $17,5 \quad(15,5-19,5)$ \\
\hline & Mehrmals & 4,4 & $(3,3-5,4)$ & $5,7 \quad(4,5-7,0)$ & $7,2 \quad(5,6-8,8)$ & $8,9 \quad(7,5-10,4)$ & 5,1 & $(3,8-6,4)$ & $5,0 \quad(3,8-6,3)$ & $7,9 \quad(6,4-9,4)$ & $10,8 \quad(9,0-12,6)$ \\
\hline \multirow[t]{2}{*}{ Milchprodukte } & Einmal & 28,0 & $(25,7-30,3)$ & $23,5(21,4-25,6)$ & $19,0(16,6-21,4)$ & $13,6(12,0-15,2)$ & 29,5 & $(27,3-31,7)$ & $20,3(18,2-22,5)$ & $15,0(12,8-17,1)$ & $12,2(10,5-14,0)$ \\
\hline & Mehrmals & 8,3 & $(6,9-9,6)$ & $5,5 \quad(4,4-6,7)$ & $4,3 \quad(3,2-5,5)$ & $4,0 \quad(2,9-5,0)$ & 6,4 & $(5,2-7,6)$ & $4,1 \quad(3,1-5,1)$ & $2,6 \quad(1,6-3,6)$ & $1,9 \quad(1,1-2,6)$ \\
\hline \multirow[t]{2}{*}{ Fleisch } & Einmal & 3,3 & $(2,3-4,4)$ & $3,5 \quad(2,6-4,4)$ & $4,6 \quad(3,4-5,8)$ & $(7,4-10,5)$ & 4,4 & $(3,3-5,5)$ & $(1,6-3,5)$ & $(2,7-5,3)$ & $(2,9-5,0)$ \\
\hline & Mehrmals & 0,9 & $(0,4-1,4)$ & $0,7 \quad(0,3-1,2)$ & $1,2 \quad(0,5-1,9)$ & $(1,4-2,8)$ & 0,4 & $(0,1-0,8)$ & $(0,2-1,1)$ & $0,6 \quad(0,2-1,1)$ & $1,0 \quad(0,5-1,5)$ \\
\hline \multirow[t]{2}{*}{ Wurst } & Einmal & 27,0 & $(24,9-29,1)$ & $31,4(29,4-33,3)$ & $27,6(24,9-30,2)$ & $27,6(25,2-29,9)$ & 26,1 & $(23,7-28,6)$ & 25,9 & $22,4(19,9-25,0)$ & $19,0(17,0-21,1)$ \\
\hline & Mehrmals & 8,3 & $(7,0-9,6)$ & $9,6 \quad(8,2-11,1)$ & $11,7(10,0-13,5)$ & $16,0(14,2-17,7)$ & 7,6 & $(6,1-9,2)$ & $7,2 \quad(6,0-8,4)$ & $8,5 \quad(6,9-10,2)$ & $9,9 \quad(8,3-11,5)$ \\
\hline \multirow[t]{2}{*}{ Cerealien } & Einmal & 21,0 & $(19,0-23,1)$ & $26,2(24,0-28,4)$ & $25,6 \quad(22,8-28,4)$ & $17,9(16,1-19,8)$ & 21,2 & $(19,1-23,3)$ & $23,3(21,0-25,6)$ & $19,4(17,2-21,6)$ & $14,0(12,3-15,8)$ \\
\hline & Mehrmals & 2,1 & $(1,3-2,8)$ & $4,2 \quad(3,3-5,2)$ & $5,0 \quad(3,8-6,2)$ & $3,1 \quad(2,1-4,0)$ & 2,4 & $(1,6-3,2)$ & $3,8 \quad(2,8-4,8)$ & $4,0 \quad(2,9-5,1)$ & $2,5 \quad(1,5-3,4)$ \\
\hline \multirow[t]{2}{*}{ Vollkornbrot } & Einmal & 19,5 & $(17,2-21,8)$ & $19,1 \quad(16,9-21,2)$ & $18,7(16,6-20,8)$ & 15,5 & 22,7 & $(20,5-24,9)$ & $17,3(15,2-19,4)$ & $17,9(15,9-20,0)$ & $20,0(17,8-22,2)$ \\
\hline & Mehrmals & 14,2 & $(12,4-16,0)$ & $15,4(13,5-17,3)$ & $15,6(13,5-17,6)$ & $15,8(14,0-17,6)$ & 14,0 & $(12,0-16,0)$ & $17,2(15,3-19,1)$ & $15,8(13,5-18,1)$ & $15,9(13,8-18,1)$ \\
\hline \multirow[t]{2}{*}{ Weißbrot } & Einmal & 26,7 & $(24,4-28,9)$ & $25,2(23,3-27,1)$ & $22,1 \quad(19,6-24,6)$ & $23,2(21,1-25,3)$ & 25,7 & $(23,5-27,9)$ & $23,7(21,4-26,0)$ & $20,9(18,2-23,5)$ & $21,4(19,3-23,5)$ \\
\hline & Mehrmals & 17,9 & $(15,7-20,2)$ & $21,9(19,7-24,1)$ & $21,5(19,0-24,1)$ & $22,7(20,4-25,0)$ & 16,8 & $(14,8-18,8)$ & $19,7(17,4-22,0)$ & $17,4(15,3-19,6)$ & $12,9(11,2-14,5)$ \\
\hline \multirow[t]{2}{*}{ Obst } & Einmal & 34,7 & $(32,2-37,2)$ & $34,0 \quad(31,8-36,2)$ & $28,4(25,7-31,1)$ & $21,9(19,8-24,1)$ & 36,6 & $(34,0-39,2)$ & $36,7(34,5-39,0)$ & $28,1 \quad(25,5-30,8)$ & $21,9(19,7-24,1)$ \\
\hline & Mehrmals & 28,8 & $(26,5-31,1)$ & $19,6(17,5-21,6)$ & $11,8 \quad(9,9-13,8)$ & $11,4 \quad(9,7-13,2)$ & 30,1 & $(27,3-32,9)$ & $23,9(21,7-26,1)$ & $21,0 \quad(18,6-23,4)$ & $21,5(19,1-23,8)$ \\
\hline Gemüse roh & Einmal & 20,8 & $(18,7-22,8)$ & $22,7(20,4-24,9)$ & $15,6(13,5-17,7)$ & $12,4(10,7-14,0)$ & 26,4 & $(24,1-28,7)$ & $24,7(22,4-27,1)$ & $20,5(17,9-23,0)$ & $19,2(17,3-21,2)$ \\
\hline & Mehrmals & 5,3 & $(4,1-6,5)$ & $4,5 \quad(3,4-5,7)$ & $3,1 \quad(2,0-4,2)$ & $2,3 \quad(1,6-3,0)$ & 5,7 & $(4,4-7,0)$ & $6,5 \quad(5,1-7,9)$ & $6,1 \quad(4,7-7,5)$ & $5,3 \quad(4,1-6,5)$ \\
\hline Kekse & Einmal & 6,3 & $(5,1-7,6)$ & $4,4 \quad(3,4-5,4)$ & $4,8 \quad(3,5-6,0)$ & $(3,1-5,2)$ & 7,5 & $(6,2-8,9)$ & $(3,2-5,2)$ & $(2,5-5,2)$ & $(2,7-4,6)$ \\
\hline & Mehrmals & 1,6 & $(1,0-2,2)$ & $0,9 \quad(0,5-1,4)$ & $0,7 \quad(0,3-1,1)$ & $(1,0-2,2)$ & 0,9 & $(0,5-1,3)$ & $0,8 \quad(0,4-1,3)$ & $2,1 \quad(1,2-2,9)$ & $1,2 \quad(0,6-1,8)$ \\
\hline Schokolade & Einmal & 13,4 & $(11,7-15,2)$ & $13,5(11,9-15,1)$ & $11,6 \quad(9,8-13,4)$ & $(8,4-11,8)$ & 14,9 & $(13,2-16,7)$ & $12,1 \quad(10,4-13,9)$ & $10,4 \quad(8,4-12,3)$ & $11,9(10,1-13,6)$ \\
\hline & Mehrmals & 3,9 & $(2,9-5,0)$ & $2,5 \quad(1,8-3,3)$ & $4,3 \quad(3,1-5,4)$ & $(3,0-4,9)$ & 3,1 & $(2,2-4,0)$ & $2,2 \quad(1,5-2,9)$ & $3,9 \quad(2,8-5,0)$ & $4,4 \quad(3,3-5,4)$ \\
\hline Süßigkeiten & Einmal & 18,6 & $(16,6-20,6)$ & $15,5(13,3-17,6)$ & $15,0 \quad(12,6-17,3)$ & $(7,9-10,8)$ & 19,8 & $(17,7-21,9)$ & $16,9(15,0-18,9)$ & $12,1(10,1-14,0)$ & $(9,2-12,3)$ \\
\hline & Mehrmals & 5,3 & $(4,1-6,5)$ & $4,7 \quad(3,6-5,8)$ & $5,6 \quad(4,3-7,0)$ & $4,6 \quad(3,5-5,6)$ & 5,0 & $(3,9-6,1)$ & $4,4 \quad(3,4-5,4)$ & $5,4 \quad(3,9-6,8)$ & $4,8 \quad(3,8-5,8)$ \\
\hline
\end{tabular}

KI) der Verzehrshäufigkeiten von ausgewählten Lebensmitteln auf Wochenbasis („weniger", ,1- bis 2-mal pro Woche“, oder „häufiger") dargestellt. Zudem sind in den - Tabellen 4 und 5 die Verzehrshäufigkeiten (und $95 \%$-KI) auf Tages- bzw. Wochenbasis für die jeweiligen Gruppen mit speziellem Migrationshintergrund und Nicht-Migranten dargestellt.

\section{Getränke}

Insgesamt trinken Jungen mit $25 \%$ statistisch signifikant häufiger mehrmals täglich Softdrinks (Erfrischungsgetränke wie Cola, Limonade) als Mädchen mit $20 \%$.
Außerdem trinken sie, zwar insgesamt selten, aber trotzdem signifikant häufiger Energydrinks (Sportler- oder Energiegetränke) als Mädchen. Diese trinken dagegen etwas häufiger täglich Leitungswasser, Mineralwasser und Früchtetee (inklusive Kräutertee), jedoch nicht statistisch signifikant. Softdrinks und Energydrinks können schnell zu einer hohen Energiezufuhr (durch den meist hohen Zuckergehalt dieser Getränke) führen und sollten daher nur selten getrunken werden. Bei Säften (Fruchtsaft, Fruchtnektar oder Gemüsesaft), Kaffee und schwarzem Tee (inklusive grüner Tee) sind kaum Unterschiede zwischen Jungen und Mädchen zu sehen
(- Tabelle 1). Der Anteil der Personen, die täglich bzw. mehrmals täglich Softdrinks zu sich nimmt, steigt signifikant mit zunehmendem Alter - etwas stärker bei Jungen als bei Mädchen ( $\bullet$ Tabelle 2). Bei den Säften ist ein gegenläufiger Trend zu sehen, hier nimmt mit dem Alter der Anteil der Jungen und Mädchen, der mehrmals täglich Saft trinkt, ab. Gleiches ist auch für Leitungswasser bei Jungen zu sehen. Der tägliche Konsum von Mineralwasser und Früchtetee (nicht dargestellt) bleibt über das Alter hinweg nahezu konstant. Schwarzer Tee und Kaffee werden in den jüngeren Altersklassen kaum getrunken. Ein wesentlicher Beitrag dieser 
Tabelle 3

Anteile mit wöchentlichem Konsum von Lebensmitteln nach Geschlecht und Altersklassen (Prozent und 95\%-Konfidenzintervall)

\begin{tabular}{|c|c|c|c|c|c|c|c|c|c|c|c|c|c|c|c|c|c|}
\hline \multirow[t]{3}{*}{ Lebensmittel } & \multirow{3}{*}{ Alter } & \multicolumn{8}{|c|}{ Jungen } & \multicolumn{8}{|c|}{ Mädchen } \\
\hline & & \multicolumn{2}{|c|}{ 3-6 Jahre } & \multicolumn{2}{|c|}{ 7-10 Jahre } & \multicolumn{2}{|c|}{ 11-13 Jahre } & \multicolumn{2}{|c|}{ 14-17 Jahre } & \multicolumn{2}{|c|}{ 3-6 Jahre } & \multicolumn{2}{|c|}{ 7-10 Jahre } & \multicolumn{2}{|c|}{ 11-13 Jahre } & \multicolumn{2}{|c|}{ 14-17 Jahre } \\
\hline & & $\%$ & (95\% KI) & $\%$ & $(95 \% \mathrm{KI})$ & $\%$ & $(95 \% \mathrm{KI})$ & $\%$ & $(95 \% \mathrm{KI})$ & $\%$ & (95\% KI) & $\%$ & $(95 \% \mathrm{KI})$ & $\%$ & $(95 \% \mathrm{KI})$ & $\%$ & $(95 \% \mathrm{KI})$ \\
\hline \multirow[t]{3}{*}{ Geflügel } & Weniger ${ }^{a}$ & 46,2 & $(42,9-49,6)$ & 48,0 & $(44,8-51,2)$ & 52,5 & $(49,2-55,7)$ & 54,1 & $(51,3-56,8)$ & 45,6 & $(42,6-48,7)$ & 48,3 & $(45,6-51,0)$ & 56,4 & $(53,2-59,6)$ & 60,1 & $(57,3-63,0)$ \\
\hline & 1- bis 2-mal & 47,4 & $(44,3-50,5)$ & 44,3 & $(41,5-47,1)$ & 38,3 & $(35,5-41,1)$ & 36,9 & $(34,5-39,2)$ & 46,1 & $(43,1-49,1)$ & 43,8 & $(41,1-46,5)$ & 35,4 & $(32,4-38,5)$ & 31,1 & $(28,3-33,8)$ \\
\hline & Häufiger ${ }^{b}$ & 6,4 & $(5,1-7,6)$ & 7,7 & $(6,3-9,1)$ & 9,2 & $(7,5-10,9)$ & 9,0 & $(7,5-10,6)$ & 8,3 & $(6,7-10,0)$ & 7,9 & $(6,3-9,5)$ & 8,2 & $(6,4-9,9)$ & 8,8 & $(7,1-10,5)$ \\
\hline \multirow[t]{3}{*}{ Fisch } & Weniger & 64,2 & $(61,4-66,9)$ & 70,3 & $(68,1-72,6)$ & 74,1 & $(71,3-76,8)$ & 77,5 & $(75,5-79,5)$ & 65,7 & $(62,9-68,4)$ & 72,6 & $(70,3-75,0)$ & 78,9 & $(76,5-81,4)$ & 82,8 & $(80,6-85,0)$ \\
\hline & 1- bis 2-mal & 34,4 & $(31,7-37,1)$ & 28,3 & $(26,0-30,5)$ & 23,5 & $(20,8-26,2)$ & 19,8 & $3(17,9-21,7)$ & 32,6 & $(30,0-35,2)$ & 26,2 & $(23,8-28,6)$ & 19,6 & $(17,2-21,9)$ & 15,8 & $(13,8-17,9)$ \\
\hline & Häufiger & 1,5 & $(0,8-2,1)$ & 1,4 & $(0,8-2,0)$ & 2,4 & $(1,7-3,2)$ & 2,7 & $(1,9-3,5)$ & 1,7 & $(1,0-2,5)$ & 1,2 & $(0,5-1,8)$ & 1,5 & $(0,8-2,2)$ & 1,3 & $(0,8-1,9)$ \\
\hline \multirow[t]{3}{*}{ Gemüse gegart } & Weniger & 29,1 & $(26,7-31,4)$ & 33,3 & $(30,8-35,7)$ & 40,5 & $(37,5-43,5)$ & 42,4 & $(39,6-45,2)$ & 28,2 & $(25,8-30,6)$ & 30,3 & $(27,6-33,0)$ & 37,3 & $(34,5-40,0)$ & 40,9 & $(37,9-43,9)$ \\
\hline & 1-bis 2-mal & 29,5 & $(27,0-32,0)$ & 32,1 & $(30,0-34,2)$ & 31,1 & $(28,2-34,0)$ & 29,8 & $(27,3-32,3)$ & 27,7 & $(25,1-30,2)$ & 31,6 & $(29,4-33,8)$ & 32,3 & $(29,3-35,2)$ & 28,0 & $(25,8-$ \\
\hline & Häufiger & 41,4 & $(38,7-44,1)$ & 34,6 & $(31,9-37,4)$ & 28,3 & $(25,6-31,1)$ & 27,8 & $(25,6-30,1)$ & 44,2 & $(41,2-47,1)$ & 38,1 & $(35,4-40,8)$ & 30,5 & $(27,8-33,1)$ & 31,1 & $(28,4-33,7)$ \\
\hline \multirow{3}{*}{$\begin{array}{l}\text { Tiefkühl- } \\
\text { gemüse }\end{array}$} & Weniger & 62,5 & $(60,0-65,1)$ & 64,8 & $(61,9-67,6)$ & 69,7 & $(67,2-72,2)$ & 71,4 & $(69,1-73,8)$ & 60,8 & $(57,9-63,7)$ & 62,4 & $(59,7-65,1)$ & 70,6 & $(67,6-73,6)$ & 72,6 & $(69,9-75,4)$ \\
\hline & 1-bis 2-mal & 26,7 & $(24,5-28,9)$ & 25,0 & $(22,7-27,2)$ & 23,4 & $(21,1-25,8)$ & 21,2 & $(19,2-23,2)$ & 27,6 & $(25,2-$ & 28,6 & $(26,1-31,1)$ & 22,4 & $(19,9$ & 20,5 & $(18,2$ \\
\hline & Häufiger & 10,7 & $(8,9-12,5)$ & 10,3 & $(8,6-12,0)$ & 6,9 & $(5,4-8,3)$ & 7,4 & $(6,0-8,7)$ & 11,6 & $(9,8-13,4)$ & 9,0 & $(7,3-10,7)$ & 7,0 & $(5,2-8,7)$ & 6,9 & $(5,6-8,2)$ \\
\hline \multirow{3}{*}{$\begin{array}{l}\text { Konserven- } \\
\text { gemüse }\end{array}$} & Weniger & 82,4 & $(80,0-84,7)$ & 82,1 & $(79,7-84,5)$ & 84,0 & $(81,7-86,3)$ & 80,5 & $(78,2-82,7)$ & 82,2 & $(80,0-84,3)$ & 81,4 & $(79,0-83,9)$ & 83,4 & $(81,1-85,6)$ & 83,0 & $(80,7-$ \\
\hline & 1-bis 2-mal & 14,9 & $(12,9-17,0)$ & 14,7 & $(12,6-16,7)$ & 13,6 & $(11,4-15,8)$ & 15,7 & $(13,6-17,7)$ & 15,3 & $(13,2-17,4)$ & 14,2 & $(12,2-16,3)$ & 12,7 & $(10,7-14,7)$ & 13,3 & $(11,4$ \\
\hline & Häufiger & 2,7 & $(1,9-3,5)$ & 3,2 & $(2,4-4,0)$ & 2,4 & $(1,6-3,3)$ & 3,9 & $(2,9-4,9)$ & 2,5 & $(1,8-3,3)$ & 4,3 & $(3,3-5,3)$ & 3,9 & $(2,8-5,0)$ & 3,7 & $(2,7-4,7)$ \\
\hline \multirow[t]{3}{*}{ Nudeln, Reis } & Weniger & 5,5 & $(4,2-6,9)$ & 7,7 & $(6,3-9,0)$ & 12,2 & $(10,3-14,0)$ & 18,4 & $(16,2-20,5)$ & 5,0 & $(3,9-6,1)$ & 6,0 & $(4,8-7,3)$ & 12,0 & $(10,0-13,9)$ & 17,4 & 9,5) \\
\hline & 1-bis 2-mal & 44,3 & $(41,0-47,7)$ & 45,0 & $(42,0-48,1)$ & 51,4 & $(48,5-54,4)$ & 46,7 & $(43,9-49,5)$ & 41,5 & $(38,4-44,5)$ & 44,4 & $(41,5-47,4)$ & 48,0 & $(45,2-50,9)$ & 42,0 & $(39,3-44,7)$ \\
\hline & Häufiger & 50,1 & $(46,5-53,8)$ & 47,3 & $(43,9-50,7)$ & 36,4 & $(33,2-39,6)$ & 34,9 & $(31,9-37,9)$ & 53,5 & $(50,2-56,9)$ & 49,5 & $(46,4-52,6)$ & 40,0 & $(36,9-43,1)$ & 40,6 & $(37,3-43,9)$ \\
\hline \multirow[t]{3}{*}{ Kartoffeln } & Weniger & 12,6 & $(10,5-14,7)$ & 13,2 & $(11,3-15,2)$ & 20,8 & $(17,7-23,8)$ & 24,3 & $(21,7-26,9)$ & 12,9 & $(11,0-14,8)$ & 11,8 & $(9,8-13,8)$ & 19,2 & $(16,6-21,7)$ & 24,0 & $(21,4-26,6)$ \\
\hline & 1- bis 2-mal & 41,3 & $(38,3-44,4)$ & 42,9 & $(40,1-45,8)$ & 42,9 & $(39,6$ & 38,4 & $(35,9-40,9)$ & 44,5 & $(41,5-47,6)$ & 42,9 & $(40,1-45,8)$ & 44,1 & $(40,5$ & 41,5 & $(38,8-$ \\
\hline & Häufiger & 46,0 & $(42,3-49,8)$ & 43,8 & $(40,3-47,3)$ & 36,3 & $(32,9-39,7)$ & 37,3 & $(34,2-40,4)$ & 42,6 & $(39,0-46,2)$ & 45,3 & $(41,8-48,7)$ & 36,8 & $(33,0-40,6)$ & 34,5 & $(31,0-38,0)$ \\
\hline \multirow[t]{3}{*}{ Pommes } & Weniger & 72,5 & $(70,0-75,0)$ & 72,2 & $(69,8-74,5)$ & 68,5 & $(65,8-71,2)$ & 65,8 & $(62,9-68,7)$ & 75,6 & $(73,1-78,1)$ & 74,0 & $(71,4-76,5)$ & 73,6 & $(70,8-76,4)$ & 76,0 & $(73,3-78,7)$ \\
\hline & 1- bis 2-mal & 24,7 & $(22,2-27,2)$ & 25,2 & $(22,9-27,4)$ & 26,0 & $(23,4-28,5)$ & 27,2 & $(24,7-29,7)$ & 22,4 & $(20,1-24,7)$ & 22,9 & $(20,6$ & 22,4 & $(19,9-24,9)$ & 20,7 & $(18$, \\
\hline & Häufiger & 2,8 & $(1,9-3,6)$ & 2,7 & $(1,9-3,5)$ & 5,5 & $(4,2-6,8)$ & 7,0 & $(5,7-8,3)$ & 2,0 & $(1,2-2,8)$ & 3,1 & $(2,1-4,2)$ & 4,0 & $(2,8-5,2)$ & 3,3 & $(2,3-4,4)$ \\
\hline \multirow[t]{3}{*}{ Fast Food } & Weniger & 90,9 & $(89,3-92,4)$ & 90,1 & $(88,5-91,7)$ & 79,9 & $(77,7-82,2)$ & 67,5 & $(65,1-70,0)$ & 92,4 & $(90,9-93,8)$ & 92,9 & $(91,5-94,3)$ & 89,7 & $(87,8-91,7)$ & 87,5 & $(85,5-89,5)$ \\
\hline & is 2 -mal & 8,4 & $(6,9-9,9)$ & 9,0 & $(7,5-10,6)$ & 16,6 & $(14,5-18,7)$ & 25,5 & $(23,2-27,7)$ & 6,3 & $(4,9-7,7)$ & 6,4 & $(5,2-7,7)$ & 8,9 & $(7,2-10,5)$ & 10,7 & $(8,8-12,6)$ \\
\hline & Häufiger & 0,8 & $(0,3-1,2)$ & 0,9 & $(0,4-1,4)$ & 3,5 & $(2,4-4,5)$ & 7,0 & $(5,6-8,4)$ & 1,3 & $(0,8-1,9)$ & 0,6 & $(0,2-1,0)$ & 1,4 & $(0,7-2,1)$ & 1,8 & $(1,1-2,5)$ \\
\hline \multirow[t]{3}{*}{ Kuchen } & Weniger & 49,0 & $(45,8-52,2)$ & 50,9 & $(48,2-53,7)$ & 54,3 & $(51,0-57,6)$ & 55,2 & $(52,3-58,0)$ & 49,9 & $(47,3-52,6)$ & 50,2 & $(47,2-53,2)$ & 56,0 & $(52,7-59,2)$ & 60,0 & $(57,2-62,8)$ \\
\hline & 1-bis 2-mal & 36,9 & $(34,1-39,8)$ & 34,4 & $(31,9-36,9)$ & 31,7 & $(28,8-34,7)$ & 29,8 & $(27,4-32,2)$ & 34,9 & $(32,6-37,3)$ & 36,7 & $(33,9-39,5)$ & 31,9 & $(29,2-34,7)$ & 27,9 & $(25,6-30,3)$ \\
\hline & Häufiger & 14,1 & $(12,2-16,0)$ & 14,7 & $(12,7-16,6)$ & 14,0 & $(12,1-15,9)$ & 15,1 & $(13,2-16,9)$ & 15,1 & $(13,2-17,1)$ & 13,1 & $(11,2-14,9)$ & 12,1 & $(10,1-14,0)$ & 12,1 & $(10,4-13,8)$ \\
\hline Knabberartikel & Weniger & 66,8 & $(64,1-69,6)$ & 65,0 & $(62,7-67,4)$ & 56,9 & $(53,6-60,3)$ & 56,0 & $(53,4-58,6)$ & 68,6 & $(66,2-71,1)$ & 67,3 & $(64,7-70,0)$ & 63,0 & $(59,7-66,2)$ & 65,6 & \\
\hline & 1-bis 2-mal & 23,4 & $(21,0-25,7)$ & 26,0 & $(23,8-28,2)$ & 27,2 & $(24,7-29,8)$ & 28,7 & $(26,6-30,8)$ & 22,3 & $(20,0-24,6)$ & 24,2 & $(22,0-26,3)$ & 24,4 & $(21,3-27,5)$ & 24,2 & $(22,0-26,5)$ \\
\hline & Häufiger & 9,8 & $(8,1-11,5)$ & 9,0 & $(7,6-10,4)$ & 15,8 & $(13,6-18,1)$ & 15,3 & $(13,5-17,1)$ & 9,0 & $(7,6-10,5)$ & 8,5 & $(7,2-9,9)$ & 12,6 & $(10,7-14,6)$ & 10,1 & $(8,4-11,8)$ \\
\hline
\end{tabular}

${ }^{a}$ weniger als einmal pro Woche, ${ }^{b}$ häufiger als 2-mal pro Woche

Getränke zur Flüssigkeitsaufnahme lässt sich erst bei den 14- bis 17-Jährigen erkennen. Generell trinken mehr Jungen und Mädchen aus Migrantenfamilien mehrmals täglich Softdrinks als NichtMigranten (• Tabelle 4). Dieser Unterschied ist jedoch nur bei türkischen und deutschstämmigen Migranten statistisch signifikant. Dagegen werden Säfte signifikant häufiger mehrmals täglich von Nicht-Migranten getrunken (Ausnahme deutschstämmige Mädchen, hier ist der Unterschied nicht signifikant). Türkische Jungen und Mädchen trinken häufiger mehrmals täglich Leitungswasser als deutschstämmige und Nicht-Migranten. Mädchen aus sonstigen Migrantenfamilien trinken ebenfalls signifikant häufiger
Leitungswasser als Nicht-Migranten. Für Mineralwasser sind keine statistisch signifikanten Unterschiede zu sehen, außer bei türkischen Mädchen, die dies häufiger als Nicht-Migrantinnen mehrmals täglich trinken (• Tabelle 4$)$.

\section{Milch und Milchprodukte}

Milch und Milchprodukte bilden neben Fleisch, Fisch und Eiern eine bedeutende Quelle für Protein und viele Vitamine. Milch ist außerdem ein Hauptlieferant von Kalzium, das für einen gesunden Knochenaufbau essenziell ist. Auch Quark, Joghurt oder Dickmilch können eine wichtige Quelle für Kalzium sein bei relativ geringer Energiedichte. Milch sollte allerdings nicht zum Durstlöschen getrunken werden, da es bei übermäßigem Konsum zu einer hohen Aufnahme von gesättigten Fettsäuren kommen kann. Zum Durstlöschen sind deshalb andere Getränke zu bevorzugen, z. B. verdünnte Fruchtsäfte und Wasser, Früchte- oder Kräutertees.

Die DGE empfiehlt täglich Milch und Milchprodukte zu konsumieren [14]. Etwa die Hälfte der Jungen (55\%) und Mädchen $(50 \%)$ trinkt täglich Milch, wobei Jungen signifikant häufiger als Mädchen mehrmals täglich Milch trinken (• Tabelle 1). Bei beiden Geschlechtern zeigt sich im täglichen Konsum ein absteigender Trend mit zunehmendem Alter. Vor allem der Anteil, der mehrmals täg- 
Tabelle 4

\section{Anteile mit täglichem Konsum von Lebensmitteln nach Geschlecht und Migrationshintergrund} (Prozent und 95\%-Konfidenzintervall)

\begin{tabular}{|c|c|c|c|c|c|c|c|c|c|c|c|c|c|}
\hline \multirow[t]{3}{*}{ Lebensmittel } & \multirow{3}{*}{$\begin{array}{l}\text { Migrations- } \\
\text { hinter- } \\
\text { grund }\end{array}$} & \multicolumn{7}{|c|}{ Jungen } & \multicolumn{5}{|c|}{ Mädchen } \\
\hline & & \multicolumn{2}{|c|}{ Türkisch } & $\begin{array}{l}\text { Deutsch- } \\
\text { stämmige }\end{array}$ & \multicolumn{2}{|c|}{$\begin{array}{l}\text { Sonstiger } \\
\text { Migrant }\end{array}$} & \multicolumn{2}{|c|}{ Nicht-Migrant } & \multicolumn{2}{|c|}{ Türkisch } & $\begin{array}{l}\text { Deutsch- } \\
\text { stämmige }\end{array}$ & $\begin{array}{l}\text { Sonstiger } \\
\text { Migrant }\end{array}$ & Nicht-Migrant \\
\hline & & $\%$ & $(95 \% \mathrm{KI})$ & (95\% KI) & $\%$ & $(95 \% \mathrm{KI})$ & $\%$ & (95\% KI) & $\%$ & $(95 \% \mathrm{KI})$ & $(95 \% \mathrm{KI})$ & $(95 \% \mathrm{KI})$ & (95\% KI) \\
\hline \multirow[t]{2}{*}{ Softdrinks } & Einmal $^{\mathrm{a}}$ & 10,5 & $(6,8-14,2)$ & $7,8 \quad(4,5-11,2)$ & 8,2 & $(5,3-11,0)$ & 8,8 & $(8,0-9,6)$ & 12,9 & $(7,7-18,1)$ & $9,1 \quad(5,6-12,7)$ & $10,7 \quad(7,3-14,1)$ & $8,1 \quad(7,3-8,9)$ \\
\hline & Mehrmals $^{\mathbf{b}}$ & 38,2 & $(32,0-44,3)$ & $31,5(25,6-37,4)$ & 29,2 & $(24,5-33,8)$ & 23,8 & $(22,6-25,0)$ & 28,7 & $(22,0-35,4)$ & $27,1 \quad(21,6-32,5)$ & $19,5(15,3-23,6)$ & $19,0 \quad(17,9-20,1)$ \\
\hline \multirow[t]{2}{*}{ Säfte } & Einmal & 15,7 & $(11,2-20,1)$ & $17,6(12,8-22,3)$ & 17,1 & $(13,2-21,0)$ & 15,2 & $(14,1-16,2)$ & 15,7 & $(10,5-20,9)$ & $12,0 \quad(8,1-16,0)$ & $(9,6-16,8)$ & $14,8 \quad(13,8-15,8)$ \\
\hline & Mehrmals & 25,3 & $(19,8-30,8)$ & $26,9(21,4-32,4)$ & 25,3 & $(20,8-29,7)$ & 36,9 & $(35,5-38,2)$ & 25,7 & $(19,0-32,4)$ & $32,2(26,3-38,0)$ & $26,6(21,8-31,4)$ & $37,3 \quad(35,9-38,7)$ \\
\hline \multirow[t]{2}{*}{ Leitungswasser } & Einmal & 12,6 & $(8,3-16,9)$ & $6,3 \quad(3,3-9,3)$ & 9,0 & $(6,0-11,9)$ & 8,3 & $(7,6-9,1)$ & 8,8 & $(4,5-13,1)$ & $5,5 \quad(2,5-8,5)$ & $(4,4-9,6)$ & $9,4 \quad(8,5-10,2)$ \\
\hline & Mehrmals & 29,0 & $(23,0-35,0)$ & $16,0(11,5-20,5)$ & 23,5 & $(19,2-27,9)$ & 19,3 & $(18,2-20,5)$ & 40,3 & $(32,9-47,7)$ & $20,6(15,5-25,8)$ & $28,1 \quad(23,1-33,1)$ & $20,9(19,7-22,1)$ \\
\hline \multirow[t]{2}{*}{ Mineralwasser } & Einmal & 10,3 & $(6,4-14,1)$ & $10,9 \quad(6,8-15,0)$ & 11,0 & $(7,7-14,2)$ & 11,3 & $(10,4-12,2)$ & 7,9 & $(4,0-11,8)$ & $10,9 \quad(7,1-14,7)$ & $7,3 \quad(4,6-9,9)$ & $11,8 \quad(10,9-12,8)$ \\
\hline & Mehrmals & 44,5 & $(38,2-50,8)$ & $42,9(36,6-49,1)$ & 44,5 & $(39,3-49,7)$ & 37,9 & $(36,6-39,3)$ & 53,2 & $(45,7-60,7)$ & $42,5(36,4-48,6)$ & $48,7 \quad(43,2-54,1)$ & $42,1 \quad(40,6-43,5)$ \\
\hline \multirow[t]{2}{*}{ Milch } & Einmal & 26,4 & $(20,8-32,0)$ & $24,6(19,2-30,0)$ & 28,8 & $(24,2-33,4)$ & 32,9 & $(31,6-34,3)$ & $21,7($ & $(15,4-27,9)$ & $19,8 \quad(14,9-24,7)$ & $33,9(28,7-39,1)$ & $32,3 \quad(31,0-33,7)$ \\
\hline & Mehrmals & 19,4 & $(14,4-24,5)$ & $23,3(18,1-28,6)$ & 21,6 & $(17,3-26,0)$ & 23,7 & $(22,5-24,8)$ & $16,6($ & $(11,2-22,0)$ & $14,8 \quad(10,5-19,1)$ & $17,9(13,7-22,0)$ & $18,5(17,4-19,6)$ \\
\hline \multirow[t]{2}{*}{ Käse } & Einmal & 27,1 & $(21,6-32,6)$ & $17,1 \quad(12,5-21,8)$ & 18,8 & $(14,8-22,8)$ & 15,4 & $(14,4-16,4)$ & 24,6 & $(18,2-31,1)$ & $15,0 \quad(10,6-19,3)$ & $16,7 \quad(12,6-20,8)$ & $16,3(15,2-17,4)$ \\
\hline & Mehrmals & 13,3 & $(9,0-17,6)$ & $9,1 \quad(5,5-12,7)$ & 8,6 & $(5,7-11,5)$ & 6,0 & $(5,4-6,7)$ & 12,1 & $(6,9-17,4)$ & $7,9 \quad(4,7-11,1)$ & $6,8 \quad(4,1-9,6)$ & $7,2 \quad(6,5-8,0)$ \\
\hline \multirow[t]{2}{*}{ Milchprodukte } & Einmal & 17,4 & $(12,7-22,1)$ & $20,3(15,1-25,4)$ & 19,4 & $(15,4-23,5)$ & 21,0 & $(19,9-22,1)$ & 16,3 & $(10,9-21,7)$ & $15,9(11,6-20,2)$ & $17,6(13,5-21,6)$ & $19,3(18,1-20,4)$ \\
\hline & Mehrmals & 6,6 & $(3,5-9,8)$ & $5,0 \quad(2,5-7,6)$ & 6,6 & $(4,2-9,1)$ & 5,4 & $(4,7-6,0)$ & 5,1 & $(2,0-8,2)$ & $2,6 \quad(0,9-4,4)$ & $6,1 \quad(3,5-8,7)$ & $3,5 \quad(3,0-4,0)$ \\
\hline \multirow[t]{2}{*}{ Fleisch } & Einmal & 5,0 & $(2,4-7,7)$ & $8,1 \quad(4,8-11,4)$ & 9,1 & $(6,2-12,0)$ & 4,9 & $(4,3-5,6)$ & 4,4 & $(1,5-7,4)$ & $(3,8-9,5)$ & $(5,4-11,5)$ & $(2,7-3,7)$ \\
\hline & Mehrmals & 2,2 & $(0,5-3,8)$ & $2,5 \quad(0,6-4,4)$ & 3,0 & $(1,3-4,7)$ & 1,0 & $(0,8-1,3)$ & 0,6 & $(0,0-1,6)$ & $(0,0-2,3)$ & $(0,0-2,4)$ & $0,7 \quad(0,4-0,9)$ \\
\hline \multirow[t]{2}{*}{ Wurst } & Einmal & 11,3 & $(7,3-15,2)$ & $29,1 \quad(23,5-34,7)$ & 21,0 & $(16,7-25,3)$ & 29,8 & $(28,5-31,1)$ & 10,6 & $(6,0-15,3)$ & $29,9(24,3-35,6)$ & $17,8(13,8-21,9)$ & $23,6 \quad(22,4-24,8)$ \\
\hline & Mehrmals & 3,8 & $(1,4-6,3)$ & $13,6 \quad(9,3-17,9)$ & 6,6 & $(4,1-9,1)$ & 12,3 & $(11,4-13,3)$ & 2,1 & $(0,0-4,2)$ & $8,8 \quad(5,3-12,2)$ & $2,1 \quad(0,6-3,5)$ & $9,1 \quad(8,3-9,9)$ \\
\hline \multirow[t]{2}{*}{ Cerealien } & Einmal & 20,9 & $(15,8-26,1)$ & $17,2 \quad(12,6-21,8)$ & 23,2 & $(18,8-27,5)$ & 22,6 & $(21,4-23,8)$ & 24,4 & $(18,0-30,9)$ & $19,9(15,1-24,8)$ & $18,1 \quad(13,9-22,3)$ & $19,0 \quad(17,8-20,1)$ \\
\hline & Mehrmals & 8,4 & $(4,7-12,1)$ & $4,1 \quad(1,6-6,6)$ & 3,6 & $(1,8-5,5)$ & 3,2 & $(2,7-3,7)$ & 7,0 & $(3,5-10,5)$ & $1,6 \quad(0,1-3,1)$ & $6,1 \quad(3,5-8,8)$ & $2,8 \quad(2,3-3,2)$ \\
\hline \multirow[t]{2}{*}{ Vollkornbrot } & Einmal & 14,2 & $(9,7-18,8)$ & $17,0(12,4-21,5)$ & 18,5 & $(14,6-22,5)$ & 18,3 & $(17,2-19,3)$ & 17,4 & $(11,3-23,5)$ & $15,7 \quad(11,1-20,3)$ & $20,4(15,9-24,9)$ & $19,9(18,7-21,0)$ \\
\hline & Mehrmals & 18,1 & $(13,2-23,1)$ & $14,8(10,5-19,2)$ & 11,0 & $(7,9-14,2)$ & 15,5 & $(14,4-16,5)$ & 15,8 & $(10,4-21,1)$ & $14,1 \quad(9,9-18,3)$ & $16,4(12,3-20,4)$ & $15,8 \quad(14,8-16,9)$ \\
\hline \multirow[t]{2}{*}{ Weißbrot } & Einmal & 23,4 & $(18,2-28,7)$ & $24,6(19,3-29,9)$ & 23,8 & $(19,5-28,1)$ & 24,4 & $(23,2-25,6)$ & 21,6 & $(15,5-27,7)$ & $28,8 \quad(23,2-34,5)$ & $25,8(21,1-30,5)$ & $22,4(21,2-23,6)$ \\
\hline & Mehrmals & 34,8 & $(28,8-40,8)$ & $26,5(21,1-31,8)$ & 24,1 & $(19,6-28,5)$ & 19,9 & $(18,7-21,0)$ & 32,0 & $(25,2-38,9)$ & $18,9(14,2-23,5)$ & $17,3(13,4-21,3)$ & $15,6(14,6-16,6)$ \\
\hline Obst & Einmal & 34,7 & $(28,6-40,8)$ & $29,9(24,3-35,5)$ & 25,5 & $(21,0-30,0)$ & 29,4 & $(28,1-30,6)$ & 30,3 & $(23,5-37,1)$ & $28,9(23,4-34,4)$ & $30,9(25,9-35,8)$ & $30,5 \quad(29,2-31,9)$ \\
\hline & Mehrmals & 17,0 & $(12,3-21,7)$ & $23,8 \quad(18,5-29,1)$ & 18,0 & $(14,0-22,0)$ & 17,5 & $(16,5-18,5)$ & 25,3 & $(18,5-32,1)$ & $26,2 \quad(20,7-31,6)$ & $22,7 \quad(18,2-27,2)$ & $23,9 \quad(22,7-25,1)$ \\
\hline Gemüse roh & Einmal & 24,2 & $(18,9-29,5)$ & $21,4(16,4-26,5)$ & 19,6 & $(15,6-23,5)$ & 17,0 & $(16,0-18,1)$ & 26,5 & $(19,8-33,1)$ & $22,6 \quad(17,4-27,8)$ & $23,0(18,4-27,6)$ & $22,4(21,2-23,6)$ \\
\hline & Mehrmals & 6,8 & $(3,7-9,9)$ & $4,2 \quad(1,6-6,8)$ & 4,6 & $(2,4-6,7)$ & 3,5 & $(3,0-4,0)$ & 8,3 & $(4,1-12,5)$ & $5,1 \quad(2,5-7,7)$ & $5,7 \quad(3,3-8,2)$ & $5,8 \quad(5,1-6,5)$ \\
\hline Kekse & Einmal & 8,9 & $(5,5-12,3)$ & $(4,4-10,6)$ & 8,1 & $(5,3-10,9)$ & 4,3 & $(3,7-4,8)$ & 2,6 & $(0,5-4,7)$ & $(3,6-9,8)$ & $(4,9-10,5)$ & $(3,9-5,0)$ \\
\hline & Mehrmals & 2,1 & $(0,5-3,7)$ & $3,2 \quad(0,8-5,6)$ & 3,4 & $(1,6-5,2)$ & 0,9 & $(0,7-1,2)$ & 3,5 & $(1,1-5,9)$ & $(1,1-5,2)$ & $(1,9-5,7)$ & $(0,5-1,1)$ \\
\hline Schokolade & Einmal & 16,4 & $(11,5-21,3)$ & $14,5(10,3-18,7)$ & 15,2 & $(11,6-18,7)$ & 11,5 & $(10,6-12,4)$ & $17,6($ & $(12,1-23,1)$ & $(8,6-16,3)$ & $(8,5-15,5)$ & $12,2(11,2-13,1)$ \\
\hline & Mehrmals & 10,8 & $(6,9-14,6)$ & $6,5 \quad(3,3-9,8)$ & 5,5 & $(3,0-8,0)$ & 3,0 & $(2,5-3,4)$ & 8,2 & $(4,2-12,1)$ & $(4,3-10,4)$ & $(3,2-8,2)$ & $2,8 \quad(2,3-3,3)$ \\
\hline Süßigkeiten & Einmal & 16,5 & $(11,8-21,1)$ & $20,1 \quad(15,0-25,2)$ & 12,7 & $(9,3-16,2)$ & 13,9 & $(12,9-14,9)$ & 16,9 & $(11,4-22,3)$ & $14,2(10,0-18,4)$ & $(9,9-17,2)$ & $14,8 \quad(13,7-15,8)$ \\
\hline & Mehrmals & 7,4 & $(3,7-11,2)$ & $9,4 \quad(5,7-13,0)$ & 8,1 & $(5,2-11,0)$ & 4,4 & $(3,8-5,0)$ & 4,9 & $(2,1-7,7)$ & $9,6 \quad(6,1-13,1)$ & $(5,2-10,9)$ & $4,3 \quad(3,7-4,9)$ \\
\hline
\end{tabular}

${ }^{a}$ einmal pro Tag, ${ }^{b}$ mehrmals pro Tag. Wegen einer unregelmäßigen Verteilung der Migranten über die Sample Points konnte bei diesen Ergebnissen das komplexe Surveydesign nicht berücksichtigt werden.

lich Milch trinkt, nimmt mit dem Alter deutlich ab (• Tabelle 2). Käse (Weich-, Schnitt- oder Hartkäse) und Frischkäse werden von Jungen und Mädchen etwa gleich oft konsumiert. Lediglich der 1bis 6-mal wöchentliche Konsum ist bei Mädchen für beide Produkte signifikant höher. Der Anteil, der mehrmals täglich (bei Jungen auch einmal täglich) Käse isst, nimmt mit dem Alter zu. Mit fast $6 \%$ essen signifikant mehr Jungen als Mädchen mehrmals täglich Milchprodukte (Quark, Joghurt oder Dickmilch). Dagegen essen signifikant mehr Mädchen 1- bis 3-mal im Monat bzw. 1- bis 6-mal wöchentlich
Milchprodukte. Die Häufigkeit des täglichen oder mehrmals täglichen Konsums dieser Produkte nimmt mit dem Alter ab.

Sowohl türkische als auch deutschstämmige Migranten trinken tendenziell seltener täglich Milch. Wird der ein- oder mehrmals tägliche Konsum zusammengefasst, dann sind diese Unterschiede im Vergleich zu den Nicht-Migranten statistisch signifikant (als Einzelkategorie lediglich der einmal tägliche Konsum bei Mädchen). Käse wird von türkischen Jungen und Mädchen statistisch signifikant häufiger einmal täglich (und tendenziell häufiger mehrmals täglich) gegessen als von anderen Kindern und Jugendlichen. Beim täglichen Konsum von Milchprodukten sind keine statistisch signifikanten Differenzen zu sehen (• Tabelle 4).

\section{Fleisch}

Nur ein geringer Teil der Studienteilnehmer isst einmal täglich Fleisch (Fleisch ohne Geflügel, Wurst, Schinken), Jungen mit 5,3\% etwas häufiger als Mädchen mit $3,7 \%$. Die Mehrheit der Jungen und Mädchen isst Fleisch lediglich 1- bis 6-mal pro Woche. Dies entspricht damit weitgehend den Empfehlungen des FKE [13] 
Tabelle 5

Anteile mit wöchentlichem Konsum von Lebensmitteln nach Geschlecht und Migrationshintergrund (Prozent und 95\%-Konfidenzintervall)

\begin{tabular}{|c|c|c|c|c|c|c|c|c|c|c|c|c|c|c|c|c|}
\hline \multirow[t]{3}{*}{ Lebensmittel } & \multirow{3}{*}{$\begin{array}{l}\text { Migrations- } \\
\text { hinter- } \\
\text { grund }\end{array}$} & \multicolumn{8}{|c|}{ Jungen } & \multicolumn{7}{|c|}{ Mädchen } \\
\hline & & \multicolumn{2}{|c|}{ Türkisch } & \multicolumn{2}{|c|}{$\begin{array}{l}\text { Deutsch- } \\
\text { stämmige }\end{array}$} & \multicolumn{2}{|c|}{$\begin{array}{l}\text { Sonstiger } \\
\text { Migrant }\end{array}$} & \multicolumn{2}{|c|}{ Nicht-Migrant } & \multicolumn{2}{|c|}{ Türkisch } & \multicolumn{2}{|c|}{$\begin{array}{l}\text { Deutsch- } \\
\text { stämmige }\end{array}$} & \multicolumn{2}{|c|}{$\begin{array}{l}\text { Sonstiger } \\
\text { Migrant }\end{array}$} & Nicht-Migrant \\
\hline & & $\%$ & $(95 \% \mathrm{KI})$ & $\%$ & $(95 \% \mathrm{KI})$ & $\%$ & $(95 \% \mathrm{KI})$ & $\%$ & $(95 \% \mathrm{KI})$ & $\%$ & $(95 \% \mathrm{KI})$ & $\%$ & $(95 \% \mathrm{KI})$ & $\%$ & $(95 \% \mathrm{KI})$ & $(95 \% \mathrm{KI})$ \\
\hline \multirow[t]{3}{*}{ Geflügel } & Weniger $^{a}$ & 34,8 & $(28,6-41,0)$ & 45,9 & $(39,6-52,2)$ & 35,7 & $(30,7-40,8)$ & 52,5 & $(51,1-53,9)$ & 46,8 & $(39,2-54,4)$ & 36,2 & $(30,3-42,2)$ & 45,6 & $(40,1-51,0)$ & $54,7 \quad(53,3-56,2)$ \\
\hline & 1-bis 2-mal & 46,2 & $(39,8-52,6)$ & 41,2 & $(35,0-47,4)$ & 43,6 & $(38,4-48,7)$ & 41,2 & $(39,8-42,6)$ & 36,7 & $(29,5-43,9)$ & 52,6 & $(46,4-58,7)$ & 36,4 & $(31,2-41,7)$ & $38,2 \quad(36,8-39,6)$ \\
\hline & Häufiger ${ }^{b}$ & 19,0 & $(14,1-23,9)$ & 12,9 & $(8,7-17,1)$ & 20,7 & $(16,5-24,9)$ & 6,3 & $(5,6-7,0)$ & 16,5 & $(10,9-22,2)$ & 11,2 & $(7,3-15,0)$ & 18,0 & $(13,9-22,1)$ & $7,1 \quad(6,3-7,9)$ \\
\hline \multirow[t]{3}{*}{ Fisch } & Weniger & 72,5 & $(67,0-78,1)$ & 67,3 & $(61,5-73,2)$ & 67,1 & $(62,3-71,9)$ & 72,4 & $(71,2-73,7)$ & 76,8 & $(70,5-83,1)$ & 70,2 & $(64,7-75,7)$ & 64,5 & $-69,8)$ & $76,5(75,3-$ \\
\hline & 1-bis 2-mal & 20,2 & $(15,3-25,1)$ & 29,6 & $(24,0-35,3)$ & 26,8 & $(22,3-31,3)$ & 26,2 & $(25,0-27,4)$ & 20,5 & $(14,4-26,6)$ & 27,7 & $(22,4-33,0)$ & 29,0 & $(24,0-34,0)$ & $22,6(21,4-23,8)$ \\
\hline & Häufiger & 7,3 & $(4,1-10,5)$ & 3,0 & $(0,9-5,2)$ & 6,1 & $(3,7-8,5)$ & 1,4 & $(1,1-1,7)$ & 2,7 & $(0,7-4,8)$ & 2,1 & $(0,3-3,9)$ & 6,5 & $(3,8-9,1)$ & $0,9 \quad(0,7-1,2)$ \\
\hline \multirow[t]{3}{*}{ Gemüse gegart } & Weniger & 48,5 & $(42,1-54,9)$ & 49,3 & $(43,1-55,6)$ & 40,0 & $(34,9-45,0)$ & 34,8 & $(33,5-36,2)$ & 44,8 & $(37,3-52,2)$ & 50,2 & $(44,0-56,4)$ & 36,5 & $(31,3-41,7)$ & $32,9(31,5-34,3)$ \\
\hline & 1-bis 2-mal & 26,6 & $(20,9-32,3)$ & 24,7 & $(19,1-30,4)$ & 24,2 & $(19,8-28,6)$ & 31,6 & $(30,3-32,9)$ & 27,4 & $(20,9-33,9)$ & 24,7 & $(19,4-30,1)$ & 24,5 & $(19,9-29,2)$ & $30,5(29$, \\
\hline & Häufiger & 24,9 & $(19,3-30,4)$ & 25,9 & $(20,4-31,5)$ & 35,8 & $(30,9-40,8)$ & 33,6 & $(32,3-34,9)$ & 27,8 & $(21,1-34,6)$ & 25,1 & $(19,8-30,5)$ & 39,0 & $(33,7-44,3)$ & $36,5 \quad(35,1-37,9)$ \\
\hline \multirow[t]{3}{*}{ Tiefkühlgemüse } & Weniger & 86,3 & $(82,0-90,6)$ & 83,1 & $(78,3-87,8)$ & 71,1 & $(66,2-76,0)$ & 65,1 & $(63,8-66,4)$ & 86,4 & $(81,4-91,5)$ & 85,9 & $(81,6-90,2)$ & 79,6 & $(75,4-83,8)$ & $64,0(62,6-65,4)$ \\
\hline & 1-bis 2-mal & 9,8 & $(6,1-13,4)$ & 13,4 & $(9,1-17,7)$ & 20,7 & $(16,3-25,1)$ & 25,5 & $(24,3-26,7)$ & 10,1 & $(5,6-14,5)$ & 10,8 & $(7,1-14,5)$ & 12,5 & $(9,2-15,9)$ & $26,9(25,6$ \\
\hline & Häufiger & 4,0 & $(1,4-6,5)$ & 3,6 & $(1,2-5,9)$ & 8,2 & $(5,2-11,2)$ & 9,4 & $(8,6-10,2)$ & 3,5 & $(1,0-6,1)$ & 3,3 & $(0,9-5,7)$ & 7,9 & $(4,9-10,8)$ & $9,1 \quad(8,3-9,9)$ \\
\hline \multirow{3}{*}{$\begin{array}{l}\text { Konserven- } \\
\text { gemüse }\end{array}$} & Weniger & 88,0 & $(83,6-92,4)$ & 84,8 & $(79,9-89,7)$ & 87,8 & $(84,4-91,3)$ & 81,1 & $(80,0-82,2)$ & 88,9 & $(84,4-93,4)$ & 86,6 & $(82,4-90,9)$ & 87,6 & $(83,9-91,2)$ & $81,6(80,5-82,7)$ \\
\hline & 1-bis 2-mal & 7,9 & $(4,2-11,6)$ & 11,3 & $(6,9-15,7)$ & 8,1 & $(5,3-10,9)$ & 15,9 & $(14,9$ & 9,2 & $(5,1-13,3)$ & 10,9 & 14,9) & 8,8 & $(5,7-11,8)$ & $14,7(13$, \\
\hline & Häufiger & 4,1 & $(1,5-6,7)$ & 3,9 & $(1,4-6,4)$ & 4,1 & $(1,9-6,2)$ & 3,0 & $(2,5-3,4)$ & 1,9 & $(0,0-3,8)$ & 2,4 & $(0,6-4,3)$ & 3,7 & $(1,5-5,8)$ & $3,7 \quad(3,2-4,2)$ \\
\hline \multirow[t]{3}{*}{ Nudeln, Reis } & Weniger & 11,0 & $(6,9-15,1)$ & 16,7 & $(12,0-21,5)$ & 10,4 & $(7,3-13,6)$ & 11,1 & $(10,2-11,9)$ & 12,3 & $(7,2-17,4)$ & 14,7 & $(10,3-19,0)$ & 11,5 & $(7,9-15,0)$ & 10,1 \\
\hline & 1-bis 2-mal & 45,0 & $(38,7-51,4)$ & 48,5 & $(42,3-54,8)$ & 37,5 & $(32,5-42,5)$ & 47,3 & $(45,9-48,7)$ & 44,4 & $(36,9-51,8)$ & 46,4 & $-52,6)$ & 36,2 & $(31,0-41,3)$ & $44,2(42$, \\
\hline & Häufiger & 44,0 & $(37,7-50,3)$ & 34,7 & $(28,9-40,6)$ & 52,1 & $(46,9-57,2)$ & 41,6 & $(40,2-43,0)$ & 43,3 & $(36,0-50,6)$ & 38,9 & $(32,9-44,9)$ & 52,4 & $(47,0-57,8)$ & $45,7(44,3-$ \\
\hline \multirow[t]{3}{*}{ Kartoffeln } & Weniger & 50,0 & $(43,6-56,3)$ & 15,5 & $(11,0-20,0)$ & 37,0 & $(32,0-42,0)$ & 15,0 & $(14,0-16,1)$ & 37,6 & $(30,4-44,8)$ & 12,5 & $(8,4-16,7)$ & 36,8 & $(31,5-42,1)$ & $15,3(14,2-$ \\
\hline & 1-bis 2-mal & 35,0 & $(29,0-41,1)$ & 41,2 & $(35,1-47,4)$ & 38,3 & $(33,3-43,3)$ & 41,7 & $(40,3-43,1)$ & 44,8 & $(37,4-52,1)$ & 49,1 & $(42,9-55,3)$ & 39,4 & $-44,7)$ & $43,0(41$, \\
\hline & Häufiger & 15,0 & $(10,6-19,4)$ & 43,3 & $(37,1-49,5)$ & 24,7 & $(20,3-29,1)$ & 43,3 & $(41,9-44,7)$ & 17,6 & $(11,7-23,5)$ & 38,4 & $(32,4-44,3)$ & 23,8 & $(19,2-28,3)$ & $41,7(40,3-43,1)$ \\
\hline \multirow[t]{3}{*}{ Pommes } & Weniger & 40,3 & $(34,0-46,5)$ & 71,2 & $(65,5-76,8)$ & 48,3 & $(43,1-53,4)$ & 72,6 & $(71,3-73,9)$ & 42,2 & $(34,8-49,6)$ & 73,4 & $(68,0-78,8)$ & 54,3 & $(48,9-59,7)$ & $78,0 \quad(76,8-79,2)$ \\
\hline & 1-bis 2-mal & 38,5 & $(32,3-44,7)$ & 25,2 & $(19,8-30,6)$ & 34,8 & $(30,0-39,7)$ & 24,5 & $(23,3-25,8)$ & 39,5 & $(32,3-46,7)$ & 24,6 & $(19,4-29,8)$ & 33,7 & $(28,6-38,7)$ & $20,2(19,0$ \\
\hline & Häufiger & 21,2 & $(15,9-26,5)$ & 3,6 & $(1,2-6,0)$ & 16,9 & $(13,0-20,9)$ & 2,9 & $(2,4-3,4)$ & 18,2 & $(12,4-24,1)$ & 2,0 & $(0,2-3,8)$ & 12,0 & $(8,5-15,5)$ & $1,8 \quad(1,4-2,2)$ \\
\hline \multirow[t]{3}{*}{ Fast Food } & Weniger & 59,9 & $(53,7-66,1)$ & 75,7 & $(70,1-81,3)$ & 69,7 & $(65,0-74,5)$ & 83,7 & $(82,7-84,8)$ & 76,3 & $(69,9-82,7)$ & 86,1 & $(81,8-90,4)$ & 84,4 & $(80,5-88,3)$ & $91,8(90,9-92,6)$ \\
\hline & & 30,3 & $(24,5-36,1)$ & 19,3 & $(14,1-24,4)$ & 23,2 & $(18,8-27,5)$ & 13,8 & $(12,8-14,8)$ & 15,9 & $(10,4-21,5)$ & 11,2 & $15,1)$ & 11,9 & $(8,3$ & $7,4 \quad(6,6$ \\
\hline & Häufiger & 9,8 & $(6,2-13,4)$ & 5,0 & $(2,1-8,0)$ & 7,1 & $(4,5-9,7)$ & 2,5 & $(2,0-2,9)$ & 7,8 & $(3,8-11,8)$ & 2,7 & $(0,7-4,7)$ & 3,7 & $(1,8-5,5)$ & $(0,6-1,1)$ \\
\hline \multirow[t]{3}{*}{ Kuchen } & Weniger & 58,6 & $(52,3-64,9)$ & 43,0 & $(36,8-49,2)$ & 52,7 & $(47,6-57,8)$ & 52,6 & $(51,2-54,0)$ & 50,3 & $(42,8-57,8)$ & 55,2 & $(49,1-61,3)$ & 60,0 & $(54,7-65,3)$ & $54,1 \quad(52,6-55,5)$ \\
\hline & 1-bis 2-mal & 27,6 & $(21,9-33,4)$ & 37,6 & $(31,5-43,7)$ & 28,9 & $(24,2-33,5)$ & 33,4 & $(32,1-34,8)$ & 32,6 & $(25,5-39,8)$ & 33,2 & $(27,4-39,0)$ & 22,8 & $(18,4-27,1)$ & $33,2(31,9-34,6)$ \\
\hline & Häufiger & 13,8 & $(9,3-18,3)$ & 19,4 & $(14,5-24,4)$ & 18,4 & $(14,5-22,3)$ & 14,0 & $(13,0-14,9)$ & 17,1 & $(11,7-22,5)$ & 11,6 & $(7,8-15,3)$ & 17,2 & $(13,1-21,3)$ & $12,7(11,8-13,6)$ \\
\hline \multirow[t]{3}{*}{ Knabberartikel } & Weniger & 29,9 & $(24,0-35,7)$ & 57,6 & $(51,5-63,8)$ & 49,4 & $(44,3-54,6)$ & 63,8 & $(62,5-65,2)$ & 32,5 & $(25,5-39,5)$ & 65,9 & $(60,1-71,7)$ & 53,5 & $(48,1-58,9)$ & $68,6(67,3-70,0)$ \\
\hline & 1-bis 2-mal & 33,7 & $(27,7-39,6)$ & 23,1 & $(17,8-28,4)$ & 30,9 & $(26,2-35,7)$ & 25,9 & $(24,6-27,1)$ & 34,2 & $(27,0-41,4)$ & 23,2 & $(18,0-28,4)$ & 28,9 & $(24,0-33,7)$ & $23,0(21,8-24,2)$ \\
\hline & Häufiger & 36,5 & $(30,3-42,6)$ & 19,2 & $(14,2-24,3)$ & 19,6 & $(15,6-23,7)$ & 10,3 & $(9,4-11,2)$ & 33,3 & $(26,4-40,2)$ & 10,9 & $(7,2-14,6)$ & 17,7 & $(13,7-21,6)$ & $8,4 \quad(7,6-9,2)$ \\
\hline
\end{tabular}

${ }^{a}$ weniger als einmal pro Woche, ${ }^{b}$ häufiger als 2-mal pro Woche; Wegen einer unregelmäßigen Verteilung der Migranten über die Sample Points konnte bei diesen Ergebnissen das komplexe Surveydesign nicht berücksichtigt werden.

und der DGE [14]. Dagegen isst jedoch ein wesentlicher Teil der Jungen (40\%) und Mädchen (32\%) täglich oder mehrmals täglich Wurst (Wurst oder Schinken). Geflügel wird von einem Großteil der Jungen und Mädchen 1- bis 6-mal pro Woche (davon meistens 1- bis 2-mal pro Woche; nicht dargestellt) konsumiert. Der Anteil, der im abgefragten Zeitraum nie Geflügel verzehrt hat, ist bei Mädchen signifikant höher als bei Jungen ( $\bullet$ Tabelle 1). Bei den 14- bis 17-jährigen Jungen ist der Anteil, der mindestens einmal täglich Fleisch konsumiert, mit $11 \%$ etwa doppelt so hoch wie bei jüngeren Jungen (gilt auch für den Konsum von 5- bis 6-mal pro Woche; nicht dargestellt). Diese plötzliche
Zunahme ist bei Mädchen im gleichen Alter nicht zu sehen. Mit steigendem Alter ist ein eher gleichmäßiger Anstieg der Anteile, die mehrmals täglich Wurst essen, zu sehen. Bei Mädchen nimmt der Anteil, der einmal täglich Wurst isst, jedoch mit steigendem Alter ab (• Tabelle 2). Der Anteil der Jungen, die häufiger als 2-mal pro Woche Geflügel konsumieren, steigt leicht mit zunehmendem Alter, jedoch nicht signifikant. Der Anteil an Jungen und Mädchen, die weniger als einmal in der Woche Geflügel konsumieren, nimmt jedoch statistisch signifikant mit dem Alter zu (• Tabelle 3$)$.

Mehr Jungen und Mädchen aus sonstigen Migrantenfamilien sowie deutsch- stämmige Migrantinnen essen einmal täglich (und tendenziell auch mehrmals täglich) Fleisch als Teilnehmer aus NichtMigrantenfamilien. Türkische Jungen und Mädchen essen statistisch signifikant seltener einmal oder mehrmals täglich Wurst als deutschstämmige und NichtMigranten, türkische Jungen außerdem weniger häufig einmal täglich als sonstige Migranten (• Tabelle 4). Ein signifikant größerer Anteil an Jungen und Mädchen aus türkischen und sonstigen Migrantenfamilien isst häufiger als 2-mal pro Woche Geflügel im Vergleich zu Nicht-Migranten (• Tabelle 5). 
Fisch

Die meisten Jungen und Mädchen essen 1- bis 3-mal im Monat Fisch (• Tabelle 1; jeweils etwa $59 \%$ ). Etwa $26 \%$ der Jungen und $23 \%$ der Mädchen essen 1- bis 2-mal pro Woche Fisch (nicht dargestellt). Diese Verzehrshäufigkeiten nehmen jedoch mit dem Alter ab, sowohl bei Jungen als auch bei Mädchen (• Tabelle 3). Der Anteil, der den empfohlenen Mindestverzehr von einmal pro Woche Fisch erreicht, nimmt mit dem Alter bei beiden Geschlechtern $\mathrm{ab}$.

Der Anteil, der häufiger als 2-mal pro Woche Fisch konsumiert, ist bei türkischen Jungen und bei Jungen und Mädchen aus sonstigen Migrantenfamilien statistisch signifikant höher als bei Nicht-Migranten. Jedoch ist der 1- bis 2mal wöchentliche Konsum am höchsten bei deutschstämmigen Migranten und bei Mädchen aus sonstigen Migrantenfamilien, sodass insgesamt bei diesen beiden Gruppen der wöchentliche Fischkonsum am häufigsten ist (• Tabelle 5).

\section{Cerealien und Brot}

Getreideprodukte wie Cerealien und Brot sollten nach DGE-Empfehlungen mehrmals täglich gegessen werden [14]. Jungen essen insgesamt häufiger Cerealien (Frühstückscerealien) und Weißbrot (Weißbrot, Graubrot oder Brötchen) als Mädchen. Weißbrot wird von etwa $45 \%$ der Jungen mindestens einmal täglich gegessen. Es wird insgesamt häufiger als Vollkornbrot (Vollkornbrot, Vollkornbrötchen oder Schwarzbrot) konsumiert, was aus ernährungsphysiologischer Sicht weniger günstig ist, da Vollkornbrot höhere Mengen an wichtigen Nährstoffen wie Vitamin B1 und B6, Magnesium, Eisen und Ballaststoffen enthält als Weißbrot. Die Häufigkeitsverteilung beim Konsum von Vollkornbrot ist bei Mädchen und Jungen relativ identisch (• Tabelle 1). Der einmal tägliche Konsum von sowohl Vollkornals auch Weißbrot nimmt mit dem Alter leicht ab, der mehrmals tägliche Konsum nimmt jedoch generell zu (Ausnahme bildet Weißbrot bei Mädchen). Beim Konsum von Vollkorn- und Weißbrot sind insgesamt keine großen Differenzen nach Alter zu beobachten. Dies lässt sich

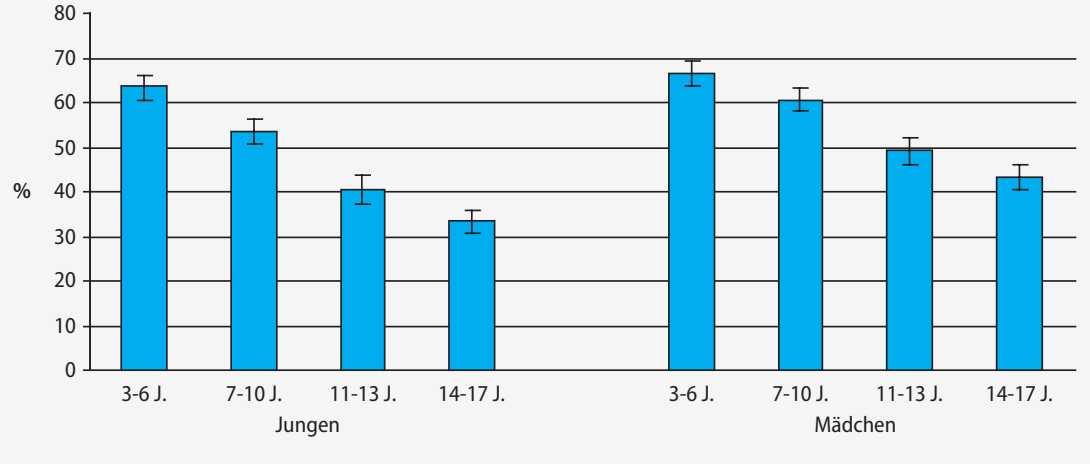

Abb. $1 \Delta$ Anteil derjenigen, die täglich Obst essen

Prävalenz und 95\%-Konfidenzintervall des täglichen Obstkonsums. Berücksichtigt wurde lediglich der Konsum von frischem Obst.

mit der typischen Mahlzeitenstruktur in Deutschland erklären, wobei bei vielen Familien Brot bei ein oder 2 Hauptmahlzeiten die Basis bildet.

Etwa $26 \%$ der Jungen isst täglich bzw. mehrmals täglich Cerealien. Am häufigsten konsumieren Jungen im Alter von 7-13 Jahren täglich Cerealien. Bei Mädchen zeigt sich eher eine Abnahme des täglichen Verzehrs von Cerealien mit dem Alter (• Tabelle 2).

Im Vergleich zu Nicht-Migranten konsumiert ein höherer Anteil türkischer Jungen und Mädchen sowie weibliche sonstige Migranten mehrmals täglich Cerealien. Beim täglichen Konsum von Vollkornbrot sind keine signifikanten, migrantenspezifischen Differenzen zu sehen. Jedoch essen türkische Jungen und Mädchen statistisch signifikant häufiger mehrmals täglich Weißbrot als sonstige und Nicht-Migranten und türkische Mädchen außerdem häufiger als deutschstämmige Migrantinnen (• Tabelle 4).

\section{Obst und Gemüse}

Obst und Gemüse gelten generell als gesunde Lebensmittel, deren Konsum sogar helfen kann, bestimmten Krebsarten vorzubeugen [26]. Seit Jahren wird z. B. mit der Kampagne „5 am Tag“ versucht, den Konsum von Obst und Gemüse zu steigern. Daher ist es erfreulich zu sehen, dass ein erheblicher Teil der Jungen (47\%) und sogar noch mehr Mädchen (55\%) mindestens einmal am Tag frisches Obst isst (siehe $\bullet$ Tabelle 2 und $\bullet$ Abb. 1). Gekochtes oder Konservenobst wird dagegen eher selten gegessen (nicht dargestellt). Der ein- oder mehrmalige tägliche Konsum von frischem Obst nimmt sowohl bei Mädchen als auch bei Jungen mit dem Alter ab (• Tabelle 2). Mehr als die Hälfte der Kinder und Jugendlichen isst 1- bis 6-mal pro Woche gegartes Gemüse (gekochtes Gemüse aus frischem Gemüse zubereitet). Tiefkühl- und Konservengemüse werden von einem Großteil der Jungen und Mädchen 1- bis 3-mal im Monat oder gar nicht konsumiert. Werden diese $3 \mathrm{Ge}$ müsezubereitungsarten zusammengefasst, essen etwa $13 \%$ der Jungen und 14\% der Mädchen täglich gegartes Gemüse. Etwa $21 \%$ der Jungen und $29 \%$ der Mädchen essen mindestens einmal am Tag rohes Gemüse (Blattsalat, Rohkost oder rohes Gemüse). Dies scheint somit für Kinder und Jugendliche die bevorzugte Gemüsevariante zu sein (• Tabelle 1). Die Anteile der Jungen und Mädchen, die häufiger als 2-mal pro Woche gegartes Gemüse oder Tiefkühlgemüse konsumieren, nimmt mit dem Alter ab. Bei Konservengemüse nimmt diese Häufigkeit leicht zu, jedoch nicht statistisch signifikant (• Tabelle 3). Schließlich nimmt auch der Verzehr von mindestens einmal täglich gegartem Gemüse mit dem Alter ab. In $\bullet$ Abb. 2 werden die Anteile der Jungen und Mädchen, die täglich Gemüse essen, dargestellt. Dies illustriert, dass auch für alle Zubereitungsarten zusammen der tägliche Konsum mit dem Alter abnimmt. Somit ist allgemein ein sinkender Obst- und Gemüsekonsum mit zunehmendem Alter erkennbar (- Tabelle 2).

Außer einem tendenziell häufigeren mehrmals täglichen Obstkonsum bei deutschstämmigen Migranten sind keine wesentlichen migrantenspezifischen Unterschiede im täglichen Obstkonsum zu 


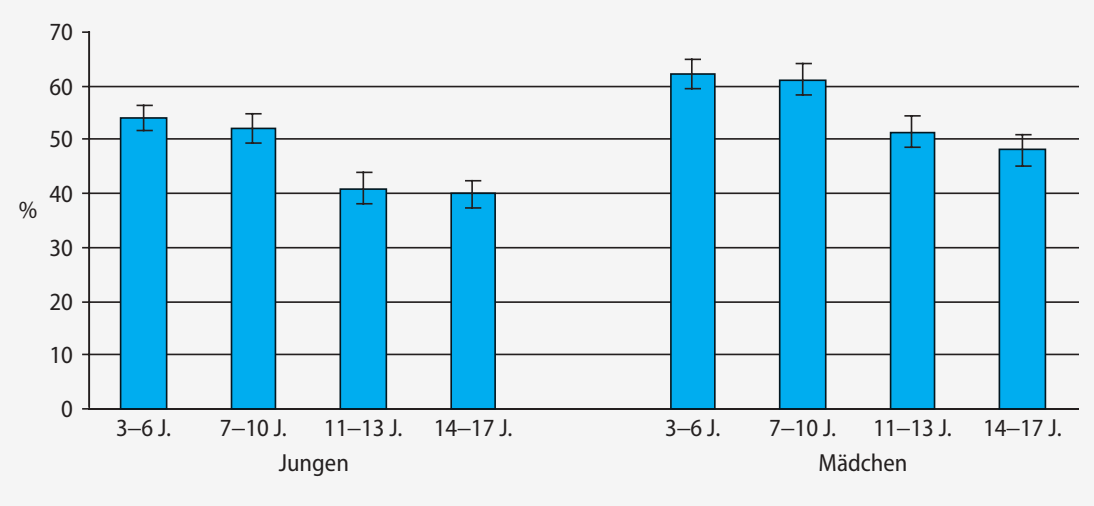

Abb. $2 \Delta$ Anteil derjenigen, die täglich Gemüse essen

Prävalenz und 95\%-Konfidenzintervall des täglichen Gemüsekonsums. Dieser setzt sich aus den Einzelvariablen gekochtes Gemüse (aus frischem Gemüse zubereitet), Tiefkühlgemüse, Konservengemüse und Blattsalat, Rohkost oder rohes Gemüse zusammen.

beobachten. Auch sind, mit Ausnahme eines statistisch signifikanten höheren Konsums von rohem Gemüse bei türkischen Jungen verglichen mit Nicht-Migranten, keine signifikanten Differenzen im täglichen Konsum vom rohem Gemüse zu sehen (• Tabelle 4). Tendenziell essen sonstige Migranten häufiger mehr als 2-mal pro Woche gegartes Gemüse (und statistisch signifikant mehr als türkische Jungen). Pro Woche wird Tiefkühlgemüse am häufigsten von Nicht-Migranten konsumiert, statistisch signifikant mehr als von türkischen und deutschstämmigen Migranten sowie sonstigen Migrantinnen. Beim Konservengemüse ist lediglich der 1- bis 2-mal wöchentliche Konsum statistisch signifikant höher bei Nicht-Migranten im Vergleich zu türkischen und sonstigen Migranten (• Tabelle 5).

\section{Nudeln, Reis, Kartoffeln}

Die Konsumhäufigkeiten von Nudeln oder Reis und von Kartoffeln (gekocht) sind bei Jungen und Mädchen etwa gleich (- Tabelle 1). Mit zunehmendem Alter nimmt bei Jungen und Mädchen der Anteil, der häufiger als 2-mal wöchentlich Nudeln oder Reis isst, ab. Gleiches gilt auch für den Kartoffelkonsum. Der Konsum von mehr als 2-mal wöchentlich Pommes (frittierten oder gebratenen Kartoffeln) nimmt jedoch (bei Mädchen nur leicht) $\mathrm{zu}(\bullet$ Tabelle 3$)$.

Über die Hälfte der sonstigen $\mathrm{Mi}$ granten isst mehr als 2-mal pro Woche Nudeln oder Reis und damit statistisch signifikant häufiger als deutschstämmige
Migranten sowie Jungen ohne Migrationshintergrund. Kartoffeln dagegen werden am meisten von deutschstämmigen sowie von Jungen und Mädchen ohne Migrationshintergrund häufiger als 2-mal pro Woche gegessen. Dafür essen türkische und sonstige Migrantenkinder häufiger als 2-mal pro Woche Pommes (• Tabelle 5).

\section{Fast Food und Knabberartikel}

Erfreulicherweise wird Fast Food (Bratwurst, Currywurst, Hamburger oder Döner Kebab) von den meisten Kindern und Jugendlichen ( $72 \%$ Jungen, $75 \%$ Mädchen) nur 1- bis 3-mal im Monat gegessen, von $9 \%$ der Jungen und $15 \%$ der Mädchen sogar nie im abgefragten Zeitraum. Jedoch essen deutlich mehr Jungen mehrmals wöchentlich Fast Food und Knabberartikel als Mädchen (• Tabelle 1). Der Anteil, der 1- bis 2-mal pro Woche oder häufiger Fast Food konsumiert, nimmt mit dem Alter bei Mädchen leicht und bei Jungen stark zu. Etwa ein Drittel der 14- bis 17jährigen Jungen isst mindestens einmal pro Woche Fast Food. Ebenso nimmt der 1- bis 2-mal wöchentliche oder häufigere Konsum von Knabberartikeln mit dem Alter zu, insbesondere bei Jungen (• Tabelle 3). Knabberartikel (Chips, Salzstangen, Cracker) werden deutlich häufiger (1- bis 2-mal pro Woche und häufiger) von türkischen Jungen und Mädchen im Vergleich zu den anderen Gruppen gegessen (- Tabelle 5).

\section{Süßigkeiten}

Täglich essen $16 \%$ der Jungen und Mädchen Schokolade und $19 \%$ der Jungen sowie $20 \%$ der Mädchen (andere) Süßigkeiten. Etwa die Hälfte der Jungen und Mädchen verzehrt lediglich 1- bis 3-mal im Monat Kuchen. Bei der Konsumhäufigkeit von Kuchen sind kaum Unterschiede zwischen Jungen und Mädchen zu sehen (• Tabelle 1). Der Anteil der Jungen und Mädchen, die weniger als einmal wöchentlich Kuchen essen, nimmt mit dem Alter zu (• Tabelle 3). Der tägliche Verzehr von Keksen, Süßigkeiten und Schokolade nimmt mit dem Alter ab (- Tabelle 2).

Mehr männliche türkische und sonstige Migranten essen einmal täglich Kekse als Nicht-Migranten (• Tabelle 4). Türkische Mädchen essen jedoch seltener als sonstige weibliche Migranten einmal täglich Kekse. Wird der ein- und mehrmals tägliche Keks- und Schokoladenkonsum zusammengefasst, dann essen Jungen ohne Migrationshintergrund seltener täglich Kekse und Schokolade als die anderen Jungen. Mädchen ohne Migrationshintergrund essen seltener täglich Kekse als deutschstämmige oder sonstige Migrantinnen und seltener täglich Schokolade als türkische Mädchen. Ein höherer Anteil der türkischen Jungen sowie der türkischen und deutschstämmigen Migrantinnen isst mehrmals täglich Schokolade als Nicht-Migranten. Jungen ohne Migrationshintergrund essen seltener als deutschstämmige Migranten täglich und seltener als sonstige Migranten mehrmals täglich Süßigkeiten. Mädchen ohne Migrationshintergrund essen seltener mehrmals täglich Süßigkeiten als deutschstämmige oder sonstige Migrantinnen. In einer Woche (1- bis 2-mal, häufiger) wird Kuchen am häufigsten von deutschstämmigen männlichen Migranten und türkischen Mädchen gegessen (• Tabelle 5).

\section{Supplemente}

In - Tabelle 6 ist die Einnahme von Multivitamintabletten (z. B. Brausetabletten) und anderen Vitamin- und Mineralstofftabletten dargestellt. Im erfragten Zeitraum nahmen etwa $72 \%$ der Jungen und $76 \%$ der Mädchen nie Multivitamintabletten. 
Tabelle 6

Einnahme von Multivitamintabletten und anderen Supplementen nach Geschlecht und Altersklassen (Prozent und 95\%-Konfidenzintervall)

\begin{tabular}{|c|c|c|c|c|c|c|c|c|c|c|c|c|c|}
\hline \multirow{3}{*}{ Geschlecht } & \multirow{3}{*}{ Alter } & \multicolumn{10}{|c|}{ Einnahmehäufigkeit Multivitamintabletten } & \multirow{2}{*}{\multicolumn{2}{|c|}{$\begin{array}{l}\text { Anteil } \\
\text { Konsumenten } \\
\text { anderer } \\
\text { Supplemente }\end{array}$}} \\
\hline & & \multicolumn{2}{|l|}{ Nie } & \multicolumn{2}{|c|}{$\begin{array}{l}\text { 1- bis 3-mal/ } \\
\text { Monat }\end{array}$} & \multicolumn{2}{|c|}{$\begin{array}{l}\text { 1- bis 6-mal/ } \\
\text { Woche }\end{array}$} & \multicolumn{2}{|c|}{ Einmal täglich } & \multicolumn{2}{|c|}{$\begin{array}{l}\text { Mehrmals } \\
\text { täglich }\end{array}$} & & \\
\hline & & $\%$ & $(95 \% \mathrm{KI})$ & $\%$ & $(95 \% \mathrm{KI})$ & $\%$ & $(95 \% \mathrm{KI})$ & $\%$ & $(95 \% \mathrm{KI})$ & $\%$ & (95\% KI) & & (95\% KI) \\
\hline \multirow[t]{4}{*}{ Jungen } & 3-6 Jahre & 78,2 & $(75,8-80,5)$ & 13,3 & $(11,5-15,3)$ & 5,5 & $(4,4-6,9)$ & 2,7 & $(1,9-3,9)$ & 0,3 & $(0,1-0,7)$ & 14,0 & $(12,1-16,2)$ \\
\hline & 7-10 Jahre & 72,7 & $(70,3-74,9)$ & 18,7 & $(16,7-20,8)$ & 5,5 & $(4,5-6,7)$ & 3,0 & $(2,3-4,0)$ & 0,1 & $(0,0-0,5)$ & 11,6 & $(10,0-13,4)$ \\
\hline & 11-13 Jahre & 69,6 & $(66,7-72,3)$ & 20,8 & $(18,5-23,4)$ & 7,7 & $(6,3-9,5)$ & 1,5 & $(1,0-2,4)$ & 0,4 & $(0,1-0,9)$ & 18,2 & $(15,7-21,0)$ \\
\hline & 14-17 Jahre & 66,6 & $(63,8-69,4)$ & 21,3 & $(19,2-23,6)$ & 8,0 & $(6,7-9,6)$ & 3,4 & $(2,6-4,5)$ & 0,6 & $(0,3-1,2)$ & 24,3 & $(21,8-27,1)$ \\
\hline \multirow[t]{4}{*}{ Mädchen } & 3-6 Jahre & 81,1 & $(78,9-83,2)$ & 11,3 & $(9,7-13,2)$ & 4,6 & $(3,6-5,7)$ & 2,8 & $(2,0-3,8)$ & 0,2 & $(0,1-0,5)$ & 12,0 & $(10,3-13,8)$ \\
\hline & 7-10 Jahre & 77,3 & $(74,8-79,5)$ & 15,4 & $(13,5-17,6)$ & 4,2 & $(3,3-5,3)$ & 2,7 & $(1,9-3,8)$ & 0,5 & $(0,2-1,1)$ & 12,7 & $(10,8-14,8)$ \\
\hline & 11-13 Jahre & 71,9 & $(69,0-74,7)$ & 20,2 & $(17,9-22,8)$ & 6,0 & $(4,7-7,8)$ & 1,7 & $(1,1-2,6)$ & 0,1 & $(0,0-0,6)$ & 18,3 & $(16,2-20,7)$ \\
\hline & 14-17 Jahre & 73,9 & $(71,5-76,1)$ & 18,3 & $(16,2-20,5)$ & 5,3 & $(4,2-6,6)$ & 2,3 & $(1,7-3,3)$ & 0,2 & $(0,1-0,6)$ & 22,5 & $(20,2-24,8)$ \\
\hline
\end{tabular}

Mit zunehmendem Alter werden häufiger Multivitamintabletten eingenommen. Etwa $13 \%$ der Jungen und $11 \%$ der Mädchen im Alter von 3-6 Jahren nehmen 1-bis 3mal im Monat Multivitamintabletten, dagegen $21 \%$ der 14 - bis 17 -jährigen Jungen und $18 \%$ der gleichaltrigen Mädchen. Der Anteil, der häufiger als 3-mal monatlich Multivitamintabletten einnimmt, liegt je nach Alter und Geschlecht etwa zwischen $8 \%$ und $12 \%$. Etwa $17 \%$ der Jungen und Mädchen haben andere Vitamin- oder Mineralstofftabletten (z. B. Vitamin C, Vitamin E, Kalzium, Eisen) eingenommen. Auch hier zeigt sich ein Alterstrend. Während bei den 3- bis 6-Jährigen $14 \%$ der Jungen und $12 \%$ der Mädchen andere Supplemente einnehmen, sind dies bei den 14 - bis 17-Jährigen etwa $24 \%$ der Jungen und $23 \%$ der Mädchen.

\section{Fertiggerichte}

- Tabelle 7 zeigt die Verzehrshäufigkeit von Fertiggerichten. Etwa 19\% der Jungen und Mädchen haben nie im erfragten Zeitraum Fertiggerichte gegessen. Insgesamt konsumieren Jungen etwas häufiger Fertiggerichte als Mädchen. Etwa $7 \%$ der 3-bis 6-jährigen Jungen und $8 \%$ der Mädchen essen 1- bis 6-mal pro Woche Fertiggerichte, wogegen statistisch signifikant mehr 14- bis 17-Jährige (33\% der Jungen und $25 \%$ der Mädchen) 1- bis 6-mal wöchentlich Fertiggerichte konsumieren.

\section{Vegetarier}

Ein eher geringer Teil der Heranwachsenden hat eine vegetarische Lebensweise. Lediglich 1,7\% der über 3-jährigen Jungen und 3,2\% der Mädchen essen kein Fleisch, Geflügel oder Wurst. Unter den 14- bis 17-Jährigen sind es immerhin 2,1\% der Jungen und 6,1\% der Mädchen. Deutlich mehr junge Vegetarier finden sich in Mittel- und Großstädten (3,2 \% bzw. 3,1\%) und unter Kindern und Jugendlichen mit Migrationshintergrund (5,3\%). Die türkischen Vegetarier machen mit 9,8\% den größten Anteil aus. Sonstige Migranten ernähren sich zu 5,3\% vegetarisch, Deutschstämmige $\mathrm{zu} 1,5 \%$ und Nicht-Migranten zu 1,9\%. Eine vegetarische Ernährung bei Kindern gilt für die Bedarfsdeckung als ausreichend, wenn die Kost ovo-lacto-vegetabil ist. Jedoch ist unter dieser Ernährungsform eine ausreichende Eisenversorgung kritisch [27].

\section{Diskussion}

Das Ernährungsverhalten ist sehr komplex und kann nicht mit nur einigen Fragen erfasst bzw. mit wenigen Zahlen umfassend dargestellt werden. Als erster Überblick werden hier die Verzehrshäufigkeiten von relevanten Lebensmittelgruppen dargestellt. Aus Platzgründen können nicht alle abgefragten Lebensmittel und Verzehrshäufigkeiten einzeln dargestellt werden.

Mit den hier dargestellten Ergebnissen kann jedoch ein erstes Bild vom aktuellen
Ernährungsverhalten bei Kindern und Jugendlichen geliefert werden. Positiv zu bewerten ist, dass relativ selten Fast Food und Fleisch gegessen und regelmäßig Milch getrunken wird (vor allem von jüngeren Kindern). Allerdings isst ein Großteil der Studienteilnehmer im Vergleich zu den Empfehlungen zu häufig Wurst, Schokolade und Süßigkeiten sowie zu selten Fisch, Gemüse und Obst. Etwa die Hälfte der Jungen und Mädchen verzehrt zwar mindestens einmal täglich Obst, jedoch ist der empfohlene mehrmals tägliche Obstkonsum selten. Gleichzeitig isst etwa die Hälfte der Teilnehmer seltener als einmal am Tag Obst. Außerdem nehmen sowohl der tägliche Obst- als auch der tägliche Gemüsekonsum mit zunehmendem Alter ab. Generell scheint Obst bei Kindern und Jugendlichen deutlich beliebter zu sein als Gemüse. Unter allen Gemüsezubereitungsarten werden Salate und rohes Gemüse bevorzugt. Brot wird insgesamt relativ häufig gegessen, jedoch scheint Weißbrot beliebter zu sein als die Vollkornvariante.

Unterschiede im Lebensmittelkonsum zeigen sich bei Jungen und Mädchen sowie nach Altersgruppen und Herkunft. Jungen trinken häufiger Milch und Softdrinks als Mädchen, diese dagegen trinken etwas häufiger Leitungs- und Mineralwasser sowie Früchte- oder Kräutertee. Jungen essen häufiger Fleisch, Wurst, Cerealien, Weißbrot und Fast Food als Mädchen. Obst und rohes Gemüse wird von Mädchen häufiger gegessen. Jünge- 
Tabelle 7

Verzehrshäufigkeit von Fertiggerichten nach Geschlecht und Altersklassen (Prozent und 95\%-Konfidenzintervall)

\begin{tabular}{|c|c|c|c|c|c|c|c|c|c|c|c|}
\hline & & \multicolumn{2}{|l|}{ Nie } & \multicolumn{2}{|c|}{ 1- bis 3-mal/Monat } & \multicolumn{2}{|c|}{ 1- bis 6-mal/Woche } & \multicolumn{2}{|c|}{ Einmal täglich } & \multicolumn{2}{|c|}{ Mehrmals täglich } \\
\hline & & $\%$ & (95\% KI) & $\%$ & (95\% KI) & $\%$ & (95\% KI) & $\%$ & (95\% KI) & $\%$ & (95\% KI) \\
\hline \multirow[t]{4}{*}{ Jungen } & 3-6 Jahre & 30,9 & $(28,5-33,4)$ & 61,5 & $(58,9-64,0)$ & 7,4 & $(6,1-9,1)$ & 0,1 & $(0,0-0,5)$ & 0,1 & $(0,0-0,5)$ \\
\hline & 7-10 Jahre & 19,5 & $(17,5-21,8)$ & 66,3 & $(63,9-68,7)$ & 14,0 & $(12,3-16,0)$ & 0,1 & $(0,0-0,5)$ & 0,0 & $(0,0-0,0)$ \\
\hline & 11-13 Jahre & 16,5 & $(14,4-18,9)$ & 61,9 & $(59,0-64,8)$ & 20,6 & $(18,3-23,1)$ & 0,9 & $(0,5-1,7)$ & 0,1 & $(0,0-0,6)$ \\
\hline & 14-17 Jahre & 11,0 & $(9,4-12,8)$ & 54,8 & $(52,1-57,5)$ & 32,6 & $(30,1-35,2)$ & 1,3 & $(0,9-2,0)$ & 0,3 & $(0,1-0,7)$ \\
\hline \multirow[t]{4}{*}{ Mädchen } & 3-6 Jahre & 30,4 & $(28,0-32,9)$ & 61,3 & $(58,5-64,0)$ & 8,0 & $(6,7-9,6)$ & 0,3 & $(0,1-0,8)$ & 0,0 & $(0,0-0,0)$ \\
\hline & 7-10 Jahre & 21,4 & $(19,1-23,8)$ & 67,6 & $(65,1-70,0)$ & 10,7 & $(9,2-12,4)$ & 0,3 & $(0,1-0,7)$ & 0,1 & $(0,0-0,4)$ \\
\hline & 11-13 Jahre & 15,3 & $(13,0-17,9)$ & 65,5 & $(62,3-68,7)$ & 18,3 & $(15,8-21,1)$ & 0,8 & $(0,3-1,7)$ & 0,1 & $(0,0-0,6)$ \\
\hline & 14-17 Jahre & 11,4 & $(9,8-13,2)$ & 62,5 & $(59,9-64,9)$ & 25,3 & $(23,2-27,5)$ & 0,8 & $(0,5-1,3)$ & 0,1 & $(0,0-0,5)$ \\
\hline
\end{tabular}

re Kinder konsumieren seltener Erfrischungsgetränke, Fleisch, Wurst und Fast Food sowie häufiger Milch, Fisch, Cerealien (nur die Mädchen), Obst, Gemüse, Schokolade, Kekse und Süßigkeiten als ältere Kinder und Jugendliche. Von den süßen Speisen abgesehen, zeigt sich somit insgesamt eher eine Verschlechterung des Ernährungsverhaltens mit zunehmendem Alter und der Trend, dass sich Mädchen ausgewogener als Jungen ernähren.

Wie erwartet, lassen sich viele Unterschiede bei den Verzehrsgewohnheiten zwischen Migranten und Nicht-Migranten beobachten. Türkische Jungen und Mädchen konsumieren am meisten täglich Leitungswasser, rohes Gemüse, aber auch frittierte oder gebratene Kartoffeln, Schokolade und Knabberartikel. Diese letzten 3 Lebensmittelgruppen gelten als eher ungesund. Bezüglich dieser Gruppen lässt sich das Ernährungsverhalten von Kindern türkischer Herkunft noch verbessern. Entgegen den Erwartungen essen türkische Kinder und Jugendliche nicht häufiger Joghurt, dafür aber häufiger Käse. Zudem essen sie seltener täglich Wurst als die anderen Gruppen. Außerdem ist auffällig, dass die sonstigen Migranten etwas häufiger Fisch, gegartes Gemüse und Nudeln oder Reis und somit häufiger Lebensmittel, die allgemein als gesund gelten, essen. Dies kann damit zusammenhängen, dass sich diese Gruppe zum großen Teil aus Personen aus dem mediterranen Raum oder Asien zusammensetzt, wo die Speisekarte deutlich mehr von diesen Lebensmitteln enthält als die traditionelle nordeuropäische Kost. Die vergleichsweise hohe Prävalenz von vegetarischen Türken könnte zum
Teil damit erklärt werden, dass die Frage hierzu nicht richtig verstanden wurde. Im Rahmen weiterer Auswertungen soll dies noch überprüft werden.

Um ein gründliches Bild über den Verzehr von Lebensmitteln zu erhalten, ist eine aufwendigere Befragungsmethodik als die Food-Frequency-Methode notwendig. In KiGGS waren etwa 2 Stunden Untersuchungszeit pro Teilnehmer angesetzt, wobei das Ernährungsverhalten lediglich einer von vielen Untersuchungsbereichen war. Daher wurde diese weniger zeitaufwendige und kostengünstige Methode eingesetzt. Die Food-Frequency-Methode gehört zu den retrospektiven Ernährungserhebungsmethoden und ermöglicht die schnelle Erfassung eines Überblicks über das Ernährungsverhalten. Die quantitative Aussagekraft ist jedoch limitiert. So dienen die erfragten Portionsmengen hauptsächlich dazu, um in Kombination mit den Verzehrshäufigkeiten die Teilnehmer in Gruppen mit einem hohen, mittleren und niedrigen Konsum der einzelnen Lebensmittel bzw. Lebensmittelkombinationen einzuteilen. Die damit errechneten Mengen sind lediglich als grobe Schätzungen zu betrachten, da unter anderem die Portionsmengen vorgegeben wurden. In Zukunft sollen die hier einzeln dargestellten Verzehrshäufigkeiten auch auf individueller Ebene kombiniert betrachtet werden, z. B. um die Vielfalt der persönlichen Ernährung zu ermitteln. Nicht der Konsum eines einzelnen Lebensmittels, sondern das gesamte Verzehrsmuster macht schließlich die individuelle Güte der Ernährung aus. Erst durch diese Kombination können die sich besonders ungünstig ernährenden Kinder und Ju- gendlichen bzw. deren Merkmale ermittelt werden.

Die KiGGS-Ergebnisse untermauern - zumindest teilweise - die bisherigen Erkenntnisse. So wurde auch in der DONALD-Studie [28], in der Kinder aus dem Raum Dortmund untersucht werden, festgestellt, dass der Konsum von gezuckerten Getränken wie Cola und Limonaden mit steigendem Alter zunimmt. Der Anteil der Kinder und Jugendlichen, der täglich Limonaden trinkt, war im Jahr 1999 in Sachsen mit $47 \%$ jedoch deutlich höher als der jetzt ermittelte Bundesdurchschnitt [29]. Kinder und Jugendliche aus Sachsen und der DONALD-Studie essen, ebenso wie die KiGGS-Teilnehmer, deutlich häufiger Obst als Gemüse. In allen Studien zeigt sich, dass Gemüse als Salat oder Rohkost bevorzugt wird. Ebenso zeigt sich sowohl bei Dortmunder Kindern und Jugendlichen als auch im KiGGS der Trend, dass mit zunehmendem Alter zwar der Konsum von Schokolade und Süßigkeiten abnimmt, dagegen häufiger Fast Food wie Hamburger und Pommes Frites gegessen wird. Wie auch im KiGGS konnte in den regionalen Untersuchungen ein regelmäßiger Konsum von Brot festgestellt werden, wobei Vollkornbrot eine eher untergeordnete Rolle spielt. In Sachsen war jedoch der Anteil der Kinder, die nie Vollkornbrot oder -brötchen isst, mit über $30 \%$ deutlich höher als im KiGGS [29]. Bei Dortmunder Kindern ist der ermittelte Anteil an Vollkornbrot am Gesamtbrotverzehr ebenfalls relativ gering [28]. Alle Untersuchungen kommen zu dem Ergebnis, dass der Konsum von Fleisch und Wurst im Vergleich zu den Empfehlungen zu hoch ist. Dies betrifft vor allem 
die Jungen. Kinder und Jugendliche aus Sachsen gaben zu etwa $50 \%$ an, wöchentlich Fisch zu essen. Dies ist deutlich mehr als im KiGGS, denn hier isst nur etwa ein Viertel der Mädchen und Jungen mindestens einmal pro Woche Fisch.

Kinder und Jugendliche sind zur Versorgung mit Nahrungsmitteln überwiegend auf ihre Eltern angewiesen [11]. Außerdem ist besonders im Vorschulalter der Bedarf an Makro- und Mikronährstoffen, bezogen auf das Körpergewicht, im Vergleich zu dem von Erwachsenen relativ hoch $[1,10]$. Darüber hinaus sind Kinder aufgrund des noch nicht vollständig ausgebildeten Immunsystems anfällig für Infektionskrankheiten, wodurch sich einerseits der Appetit verringern kann, andererseits jedoch der Bedarf an bestimmten Nährstoffen erhöht ist [10].

Die Entwicklung zahlreicher Körperfunktionen während der Wachstumsphase und die erheblichen körperlichen Veränderungen innerhalb weniger Jahre [30] erfordern eine ausgewogene und bedarfsgerechte Versorgung mit Nährstoffen entsprechend den Referenzwerten [31, 32]. Unterernährung und Nährstoffmangel im Kindesalter können langfristige Folgen für Ernährungsstatus, Wachstum, Gesundheit und kognitive Fähigkeiten haben [33].

Jedoch kann auch eine Überversorgung in frühen Jahren weitreichende Konsequenzen haben. Fehlernährung und Übergewicht werden bereits im $\mathrm{Zu}$ sammenhang mit chronischen Erkrankungen im Kindes- und Jugendalter, wie z.B. Adipositas, Diabetes mellitus und Bluthochdruck, diskutiert $[1,34,35]$. Da die sensorischen Vorlieben schon früh geprägt werden können, ergibt sich daraus ein weiterer Grund, Kindern schon zeitig eine gesunde Ernährungsweise (mit unter anderem reichlich Obst und Gemüse) näherzubringen. Kinder gewöhnen sich an die Lebensmittel, die sie regelmäßig essen, und den dazu gehörigen Geschmack. Diese Präferenzen für bestimmte Lebensmittel bleiben oft bis zum Erwachsenenalter bestehen [36]. Außerdem beeinflusst das Ernährungsverhalten der Eltern und des sozialen Umfelds (Lehrer, Freundeskreis, Medien) maßgeblich die Ernährungsgewohnheiten des Kindes [1, 36, 37]. Zum Schutz der Gesundheit ist eine adäquate und gesunde Ernährungsweise somit so früh wie möglich anzustreben.

\section{Ausblick}

Es wurde hier noch keine Kombination der Verzehrshäufigkeiten mit den Verzehrsmengen vorgenommen, da die vorgegebenen Antwortmöglichkeiten und Portionen im FFQ relativ grob sind und somit die quantitative Aussagekraft eingeschränkt ist. In weiteren Auswertungen können die Verzehrsmengen jedoch herangezogen werden, um Gruppenvergleiche durchzuführen, z. B. nach sozialem Status oder Herkunft. Weiter sind Ernährungsindizes in Entwicklung, die es ermöglichen, die Ernährung als Ganzes auf individuellem Niveau sowie im Gruppenvergleich zu betrachten. Jedoch wird dies immer noch nicht der Multidimensionalität der Ernährung gerecht, weshalb in einem weiteren Schritt Ernährungsmuster analysiert werden sollen. Auch wird der Zusammenhang zwischen Ernährungsbzw. Gesundheitsverhalten und Übergewicht näher untersucht. Wesentlich quantitativere Aussagen zur Ernährungsweise können mit den Daten aus EsKiMo [16, 17], die eine Unterstichprobe vom KiGGS bilden, gemacht werden.

\section{Korrespondierender Autor}

\section{Dr. Gert B. M. Mensink}

Robert Koch-Institut, Abteilung für Epidemiologie und Gesundheitsberichterstattung

Postfach 650261

13302 Berlin, BRD,

E-Mail: MensinkG@rki.de

\section{Literatur}

1. Alexy U, Kersting M (1999) Was Kinder essen - und was sie essen sollten. Hans Marseille Verlag, München

2. Karg G, Gedrich K (2000) Ernährungssituation in Deutschland. In: Deutsche Gesellschaft für Ernährung (Hrsg) Ernährungsbericht 2000. Eigenverlag, Frankfurt, S 17-80

3. Pudel V, Borchardt A, Ellrott T et al. (2000) Essverhalten und Ernährungszustand von Kindern und Jugendlichen - eine Repräsentativerhebung in Deutschland. In: Deutsche Gesellschaft für Ernährung (Hrsg) Ernährungsbericht 2000. Eigenverlag, Frankfurt, S 115-146
4. Schöch G, Clausen K, Sichert-Hellert W, Kersting M (2000) Ernährungssituation in Kindertagesstätten Die Kindertagesstätten-Ernährungs-SituationsStudie „KESS". In: Deutsche Gesellschaft für Ernährung (Hrsg) Ernährungsbericht 2000. Eigenverlag, Frankfurt, S 97-114

5. Merx H, Reuter M, Winkler G (2003) Ernährungssituation von Kindern. Ergebnisse einer Erhebung bei Erstklässlern im Rems-Murr-Kreis. ErnährungsUmschau 50:376-382

6. Ravens-Sieberer U, Thomas C (2003) Gesundheitsverhalten von Schülern in Berlin. Ergebnisse der HBSC-Jugendgesundheitsstudie 2002 im Auftrag der WHO. Robert Koch-Institut, Berlin, S 52-57

7. AdolfT, Schneider R, Eberhardt W et al. (1995) Ergebnisse der Nationalen Verzehrsstudie (19851988) über die Lebensmittel- und Nährstoffaufnahme in der Bundesrepublik Deutschland. In: Kühler W, Anders HJ, Heeschen W (Hrsg) VERASchriftenreihe, Band XI. Fleck-Verlag, Niederkleen

8. Brombach C, Wagner U, Eisinger-Watzl M, Heyer A (2006) Die Nationale Verzehrsstudie II. Ziel: Aktuelle und belastbare Primärdaten für die Ernährungsberichterstattung des Bundes generieren. Ernährungs-Umschau 53:4-9

9. Heseker H, Oepping A, Vohmann C (2003) Verzehrsstudie zur Ermittlung der Lebensmittelaufnahme von Säuglingen und Kleinkindern für die Abschätzung eines akuten Toxizitätsrisikos durch Rückstände von Pflanzenschutzmitteln (VELS). Forschungsbericht der Universität Paderborn im Auftrag des Bundesministeriums für Verbraucherschutz, Ernährung und Landwirtschaft, Bonn

10. Poskitt EM (1998) Nutritional problems of preschool children. In: Sadler MJ, Strain JJ, Caballero $B$ (eds) Encyclopedia of human nutrition, Volume One. Academic Press, San Diego London, pp 340-349

11. Tuttle C (1999) Childhood and adolescence. In: Mann J, Truswell AS (eds) Essentials of human nutrition. Oxford University Press, Oxford New York Tokyo, pp 481-487

12. Westenhöfer J (2002) Establishing dietary habits during childhood for long-term weight control. Ann Nutr Metab 46 [Suppl 1]:18-23

13. Forschungsinstitut für Kinderernährung Dortmund (2005) optimiX. Empfehlungen für die Ernährung von Kindern und Jugendlichen. 5. überarbeitete Auflage. Forschungsinstitut für Kinderernährung, Dortmund

14. Deutsche Gesellschaft für Ernährung (2004) 10 Regeln für eine vollwertige Ernährung. Eigenverlag, Bonn

15. Infodienst Verbraucherschutz - Ernährung - Landwirtschaft (2006) Die aid-Ernährungspyramide. http://www.aid.de/ernaehrung/ernaehrungs pyramide.php

16. Bauch A, Mensink GBM, Vohmann C et al. (2006) EsKiMo. Die Ernährungsstudie bei Kindern und Jugendlichen. Ernährungs-Umschau 53:380-385

17. Mensink GBM, Richter A, Vohmann C et al. (2007) EsKiMo - Das Ernährungsmodul im Kinder- und Jugendgesundheitssurvey (KiGGS). Bundesgesundheitsbl Gesundheitsforsch Gesundheitsschutz 50:902-908

18. Kurth B-M (2007) Der Kinder- und Jugendgesundheitssurvey (KiGGS): Ein Überblick über Planung, Durchführung und Ergebnisse unter Berücksichtigung von Aspekten eines Qualitätsmanagements. Bundesgesundheitsbl Gesundheitsforsch Gesundheitsschutz 50:533-546 
19. Kamtsiuris $P$, Lange M, Schaffrath Rosario A (2007) Der Kinder- und Jugendgesundheitssurvey (KiGGS): Stichprobendesign, Response und Nonresponse-Analyse. Bundesgesundheitsbl Gesundheitsforsch Gesundheitsschutz 50:547-556

20. Hölling H, Kamtsiuris $P$, Lange $M$ et al. (2007) Der Kinder- und Jugendgesundheitssurvey (KiGGS): Studienmanagement und Durchführung der Feldarbeit. Bundesgesundheitsbl Gesundheitsforsch Gesundheitsschutz 50:557-566

21. SPSS Inc. (2005) SPSS 14.0 Base Benutzerhandbuch. SPSS Inc. Chicago

22. SAS Institute Inc. (2004). SAS/STAT 9.1 User's Guide. Cary, NC: SAS Institute Inc.

23. Thefeld W, Bergmann KE, Burger M et al. (2002) Der Kinder- und Jugendgesundheitssurvey: Ermittlung des Gesundheitsverhaltens von Eltern und Kindern. Gesundheitswesen 64 [Sonderheft 1]:S36-S42

24. Mensink G, Burger M (2004) Was isst du? Ein Verzehrsfragebogen für Kinder und Jugendliche. Bundesgesundheitsbl Gesundheitsforsch Gesundheitsschutz 47:219-226

25. Schenk L, Ellert U, Neuhauser H (2007) Kinder und Jugendliche mit Migrationshintergrund in Deutschland. Methodische Aspekte im Kinderund Jugendgesundheitssurvey (KiGGS). Bundesgesundheitsbl Gesundheitsforsch Gesundheitsschutz 50:590-599

26. Key TJ, Schatzkin A, Willett WC et al. (2004) Diet, nutrition and the prevention of cancer. Public Health Nutrition 7(1A):187-200

27. Lentze MJ (2001) Vegetarische Ernährung und Außenseiterdiäten im Kindesalter. Monatsschr Kinderheilkd 149:19-24

28. Kersting M, Alexy U, Kroke A, Lentze MJ (2004) Kinderernährung in Deutschland. Ergebnisse der DONALD-Studie. Bundesgesundheitsbl Gesundheitsforsch Gesundheitsschutz 47:213-218

29. Sächsisches Staatsministerium für Umwelt und Landwirtschaft (2001) 1. Sächsische Verzehrsstudie. Ergebnisse - Daten - Auswertung. Eigenverlag, Dresden

30. Caballero B (1998) Nutritional requirements of school children. In: Sadler MJ, Strain JJ, Caballero $B$ (eds) Encyclopedia of human nutrition, Volume One. Academic Press, San Diego London, pp 350-352

31. Deutsche Gesellschaft für Ernährung, Österreichische Gesellschaft für Ernährung, Schweizerische Gesellschaft für Ernährungsforschung, Schweizerische Vereinigung für Ernährung (2000) Referenzwerte für die Nährstoffzufuhr. Umschau Verlag, Frankfurt am Main.

32. Heine W (1999) Ernährung vom Säuglings- bis zum Jugendalter. In: Biesalski HK, Fürst P, Kasper H et al. (Hrsg) Ernähungsmedizin. Georg Thieme, Stuttgart New York, S 201-211

33. Nelson M (1998) Nutritional problems of preschoo children. In: Sadler MJ, Strain JJ, Caballero B (eds) Encyclopedia of human nutrition, Volume One. Academic Press, San Diego London, pp 352-356

34. Wilkin TJ, Voss LD, Metcalf BS et al. (2004) Metabolic risk in early childhood: the Early Bird Study. Int J Obes 28:S64-S69

35. Couch SC, Daniels SR (2005) Diet and blood pressure in children. Curr Opin Pediatr 17: 642-647

36. Leach H (1999) Food habits. In: Mann J, Truswell AS (eds) Essentials of human nutrition. Oxford University Press, Oxford New York Tokyo, pp 515-521

37. Ramos M, Stein LM (2000) Development children's eating behaviour. J Pediatr 76 [Suppl 3]:

S229-S237

\section{Direkter Draht zwischen Gehirn und Immunsystem}

Bei Mäusen sind zahlreiche Immunzellen im Gewebe rund um den Darm unmittelbar mit Nervenfasern und -zellen verknüpft. Das ergaben mikroskopische Untersuchungen von Wissenschaftlern am Helmholtz-Zentrum für Infektionsforschung.

Für ihre Studie verwendeten die Forscher aus Braunschweig weiterentwickelte immunhistochemische Methoden. Dadurch konnten sie bis zu 7 Zelltypen gleichzeitig in histologischen Schnitten charakterisieren und zahlreiche Kontakte zwischen Nerven- und Immunzellen sichtbar machen. Dazu passt der Befund, dass in Lymphknoten rund um den Darm wie etwa den Peyerschen Plaques etliche Nervenfasern enden. Die Forscher fanden außerdem Hinweise darauf, dass Immunzellen die Transmitter des Nervensystems wahrnehmen können.

Die Wissenschaftler vermuten, dass die Verhältnisse beim Menschen nicht viel anders als bei der Maus liegen: Auch hier scheinen Gehirn und Psyche auf das Immunsystem zu wirken. Für viele Infektions- und Autoimmunerkrankungen beispielsweise ist im klinischen Alltag ein Zusammenhang zwischen der Psyche und der Schwere der Krankheit bekannt. Wie das Nervensystem die Immunabwehr im Einzelnen steuert, bleibt allerdings noch unklar. Die Forscher erhoffen sich von ihren Ergebnissen aber in naher Zukunft Aufschluss über bestimmte Infektionsprozesse. So könnten Krankheitserreger wie Prionen, die über den Darm in das Nervensystem gelangen, den Weg über die Lymphknoten des Darms nehmen - und dabei die beschriebenen Verbindungen zwischen Nerven- und Lymphgefäßen nutzen.

Literatur: Ma B, von Wasiliewski R, Lindenmaier W, Dittmar KE (2007) Immmunohistochemical study of the blood and lymphatic vasculature and the innervation of mouse gut and gut-associated lymphoid tissue. Anat Histol Embryol 36(1):62-74

Helmholtz-Zentrum für Infektionsforschung, www.gbf.de 